Article

\title{
Functional Analysis of P450 Monooxygenase SrrO in the Biosynthesis of Butenolide-Type Signaling Molecules in Streptomyces rochei
}

\author{
Aiko Teshima ${ }^{1}$, Nozomi Hadae ${ }^{1}$, Naoto Tsuda ${ }^{1}$ and Kenji Arakawa ${ }^{1,2, *(D)}$ \\ 1 Department of Molecular Biotechnology, Graduate School of Advanced Sciences of Matter, Hiroshima \\ University, 1-3-1 Kagamiyama, Higashi-Hiroshima, Hiroshima 739-8530, Japan; \\ teshima-ai@hiroshima-u.ac.jp (A.T.); aaeooi2@gmail.com (N.H.); naoto.tsuda@ncx.nagase.co.jp (N.T.) \\ 2 Unit of Biotechnology, Graduate School of Integrated Sciences for Life, Hiroshima University, \\ 1-3-1 Kagamiyama, Higashi-Hiroshima, Hiroshima 739-8530, Japan \\ * Correspondence: karakawa@hiroshima-u.ac.jp; Tel./Fax: +81-82-424-7767
}

Received: 31 July 2020; Accepted: 24 August 2020; Published: 25 August 2020

\begin{abstract}
Streptomyces rochei 7434AN4 produces two structurally unrelated polyketide antibiotics lankacidin and lankamycin, and their biosynthesis is tightly controlled by butenolide-type signaling molecules SRB1 and SRB2. SRBs are synthesized by SRB synthase SrrX, and induce lankacidin and lankamycin production at $40 \mathrm{nM}$ concentration. We here investigated the role of a P450 monooxygenase gene $\mathrm{srrO}$ (orf84), which is located adjacent to $\operatorname{srrX}$ (orf85), in SRB biosynthesis. An srrO mutant KA54 accumulated lankacidin and lankamycin at a normal level when compared with the parent strain. To elucidate the chemical structures of the signaling molecules accumulated in KA54 (termed as KA54-SRBs), this mutant was cultured (30 L) and the active components were purified. Two active components (KA54-SRB1 and KA54-SRB2) were detected in ESI-MS and chiral HPLC analysis. The molecular formulae for KA54-SRB1 and KA54-SRB2 are $\mathrm{C}_{15} \mathrm{H}_{26} \mathrm{O}_{4}$ and $\mathrm{C}_{16} \mathrm{H}_{28} \mathrm{O}_{4}$, whose values are one oxygen smaller and two hydrogen larger when compared with those for SRB1 and SRB2, respectively. Based on extensive NMR analysis, the signaling molecules in KA54 were determined to be $6^{\prime}$-deoxo-SRB1 and $6^{\prime}$-deoxo-SRB2. Gel shift analysis indicated that a ligand affinity of $6^{\prime}$-deoxo-SRB1 to the specific receptor SrrA was 100-fold less than that of SRB1. We performed bioconversion of the synthetic $6^{\prime}$-deoxo-SRB1 in the Streptomyces lividans recombinant carrying SrrO-expression plasmid. Substrate $6^{\prime}$-deoxo-SRB1 was converted through $6^{\prime}$-deoxo-6'-hydroxy-SRB1 to SRB1 in a time-dependent manner. Thus, these results clearly indicated that SrrO catalyzes the C-6' oxidation at a final step in SRB biosynthesis.
\end{abstract}

Keywords: Streptomyces; signaling molecule; biosynthesis; cytochrome P450; secondary metabolite

\section{Introduction}

The soil-dwelling filamentous bacterial genus, Streptomyces, is well characterized by their distinct properties to produce a vast array of pharmaceutically and agriculturally important secondary metabolites. Streptomyces species generally produce diffusible signaling molecules that induce secondary metabolite production and morphological differentiation [1,2]. Hitherto discovered signaling molecules are classified into three groups; $\gamma$-butyrolactone-type, furan-type, and butenolide-type [3-6]. For example, $\gamma$-butyrolactone-type contains A-factor in Streptomyces griseus for streptomycin production $[7,8]$, furan-type does methylenomycin furans in Streptomyces coelicolor for methylenomycin production [9], and butenolide-type contains avenolide in Streptomyces avermitilis for avermectin [10] and SRBs in Streptomyces rochei for lankacidin/lankamycin [11]. 
Streptomyces rochei strain 7434AN4 produces two structurally unrelated polyketide antibiotics, lankacidin and lankamycin (Figure S1), and carries three linear plasmids (pSLA2-L, -M, and -S) [12]. Lankacidin and lankamycin inhibit peptide synthesis synergistically by targeting neighboring sites in bacterial ribosome [13,14]. In addition, lankacidins could exhibit considerable antitumor activity in paclitaxel-like action $[15,16]$. These biosynthetic gene clusters are located on the largest giant linear plasmid pSLA2-L (210,614 bp) [17-20]. In addition, their regulatory genes including an SRB biosynthesis gene ( $\operatorname{sr} X)$, an SRB receptor gene ( $s r A$ ), and two SARP (Streptomyces antibiotic regulatory protein) genes ( $s r r Y$ and $s r r Z$ ) are coded on pSLA2-L. We revealed that the signaling pathway goes from $s r r X$ through $s r r A$ to $\operatorname{srr} Y$, leading to lankacidin production [21,22]. For lankamycin production, $\operatorname{srr} Y$ directly activates the transcription of $\operatorname{sr} Z$ [23]. In addition, we have elucidated the chemical structures of signaling molecules SRB1 and SRB2 in S. rochei; SRB1 was determined to be 2-(1'-hydroxyl-6'-oxo-8'-methylnonyl)-3-methyl-4-hydroxybut-2-en-1,4-olide $\left(\mathrm{C}_{15} \mathrm{H}_{24} \mathrm{O}_{5}\right)$, while SRB2 was 2-(1'-hydroxyl-6'-oxo-8'-methyldecyl)-3-methyl-4-hydroxybut-2-en-1,4-olide $\left(\mathrm{C}_{16} \mathrm{H}_{26} \mathrm{O}_{5}\right)$ (Figure 1) [11]. Their $\mathrm{C}^{\prime}$ ' stereochemistry was determined to be $R$ based on chiral HPLC analysis. It is noteworthy that several possible genes for SRB biosynthesis were found around $\operatorname{srr} X$ (orf85) on pSLA2-L; an NAD-dependent dehydrogenase gene $\operatorname{sr} G$ (orf81), a phosphatase gene $s r r P$ (orf83), a P450 monooxygenase gene $\mathrm{srrO}$ (orf84), and a thioesterase gene $\mathrm{srrH}$ (orf86).

A<smiles></smiles>

B<smiles>Cc1[Z6](O)oc(=O)c1[C@@H](O)CCCCCCC(C)C</smiles><smiles>CC[C@H](C)CC(=O)CCCC[C@@H](O)C1=C(C)[Z9](O)=CC1=O</smiles><smiles>CC[C@H](C)CCCCCC[C@@H](O)c1c(C)[Z6](O)oc1=O</smiles>

Figure 1. Structures of Streptomyces rochei signaling molecules. (A) natural signaling molecules SRB1 and SRB2 isolated from S. rochei 7434AN4; (B) 6'-deoxo-SRB1 (1) and 6'-deoxo-SRB2 (2) isolated from the $\mathrm{srrO}$ mutant (strain KA54) of $S$. rochei.

The cytochrome P450 comprises a ferric heme-containing enzyme superfamily that catalyzes the oxygenation reaction on vast array of substrates including antibiotics, lipids, and steroids, and is widely distributed across the Kingdoms from bacteria to human [24-26]. One of the typical chemical reactions catalyzed by $\mathrm{P} 450$ enzymes is an oxidation of non-reactive $\mathrm{C}-\mathrm{H}$ bonds [27]. Monooxygenase reactions are involved in the macrolide biosynthesis of erythromycin [28,29], oleandomycin [30], and lankamycin [19]. Location of $s r r O$ (orf84) gene at the neighbor of $\mathrm{srrX}$ (orf85) indicates that $\mathrm{srrO}$ may be involved in SRB biosynthesis in S. rochei. We here analyzed the function of $s r r O$ by gene inactivation and enzymatic bioconversion experiments, the results of which will be described in this paper.

\section{Materials and Methods}

\subsection{Strains, Reagents, and Culture Conditions}

All strains and plasmids used in this study were listed in Table S1. Strain 51252 that harbors pSLA2-L in addition to the chromosome was used as a parent strain [12]. Strain KA20, a double mutant of $\operatorname{srr} X$ and the transcriptional repressor gene $\operatorname{srr} B$, was used as signaling-molecule indicator strain $[11,21]$. YM medium $(0.4 \%$ yeast extract, $1.0 \%$ malt extract, and $0.4 \%$ D-glucose, $\mathrm{pH} 7.3)$ was 
used for signaling-molecule synthesis and bioassay. For protoplast preparation and SrrO-protein expression, Streptomyces strains were grown in YEME liquid medium [31]. Protoplasts were regenerated on R1M solid medium [32]. To construct targeting plasmids for srrO mutation, E. coli strains were grown in Luria-Bertani medium supplemented with ampicillin $(100 \mu \mathrm{g} / \mathrm{mL})$ when necessary. Genetic manipulations for Streptomyces [31] and E. coli [33] were performed according to the described procedures.

\subsection{Spectroscopic Instruments}

NMR spectra were recorded on ECA-500 and/or ECA-600 spectrometers (JEOL, Tokyo, Japan) equipped with a field gradient accessory. Deuteriochloroform (99.8 atom\% enriched; Kanto Chemical Co., Ltd., Tokyo, Japan) was used as a solvent. Chemical shifts were recorded as a $\delta$ value based on a resident solvent signal $\left(\delta_{C}=77.0\right)$, or an internal standard signal of tetramethylsilane $\left(\delta_{\mathrm{H}}=0\right)$. High resolution electrospray ionization (ESI) mass spectra were measured by a LTQ Orbitrap XL mass spectrometer (Thermo Fisher Scientific, Waltham, MA, USA). High resolution gas chromatography-time of flight mass spectra (ionization mode; CI) were acquired on a JMS-T100 GCV 4G (JEOL, Tokyo, Japan). Optical rotations were measured using a DIP-370 polarimeter (JASCO Cooperation, Tokyo, Japan). IR spectra were recorded on a IRAffiniy-1 spectrometer (Shimadzu Cooperation, Kyoto, Japan) using the ATR (Attenuated Total Reflection) method.

\subsection{Construction of the srrO Mutant KA54}

A 3.8-kb StuI-EcoRI fragment (nt 143,844,147,674 of pSLA2-L) containing an srrO gene was ligated with 2.8-kb StuI-EcoRI fragment of Litmus 28i to give pKAR3041. To a ClaI restriction site at the 5'-terminal region of srrO was introduced a 1.0-kb ClaI fragment of aac(3)IV gene cassette conferring apramycin resistance. The resulting plasmid pKAR3043 was digested with EcoRI and StuI, and the vector of which was replaced with an EcoRI-SmaI fragment of pRES18, an E. coli-Streptomyces shuttle vector [34], to afford pKAR3044. This plasmid was transformed into protoplast of strain 51252, and then an srrO mutant KA54 was obtained through homologous recombination according to our protocol [18] (Figure S2).

\subsection{Metabolites in the srrO Mutant KA54}

Antibiotic production in strain KA54 was analyzed by high performance liquid chromatography (HPLC) and thin layer chromatography (TLC) in comparison with that in parent strain 51252. The crude extract was applied on a COSMOSIL CHOLESTER column $(4.6 \times 250 \mathrm{~mm}$, Nacalai Tesque, Kyoto, Japan) and eluted with a mixture of acetonitrile-10 mM sodium phosphate buffer $(\mathrm{pH} 8.2)(3: 7, v / v)$ at a flow rate of $1.0 \mathrm{~mL} / \mathrm{min}$. The eluate was monitored at $230 \mathrm{~nm}$ with a MD-2010 multiwavelength photodiode array detector (JASCO Cooperation, Tokyo, Japan). TLC was developed with a mixture of $\mathrm{CHCl}_{3}$-methanol $(15: 1, v / v)$ and baked after spraying with anisaldehyde- $\mathrm{H}_{2} \mathrm{SO}_{4}$.

\subsection{Isolation of Signaling Molecules from the srrO Mutant KA54}

Seed culture $(20 \mathrm{~mL})$ of strain KA54 was inoculated into to $2 \mathrm{~L}$ YM medium in 5-L Erlenmeyer flask, which was grown at $28^{\circ} \mathrm{C}$ for $36 \mathrm{~h}$. A total of $30 \mathrm{~L}$ culture broth was extracted with equal volume of EtOAc twice. The combined organic phase was dried $\left(\mathrm{Na}_{2} \mathrm{SO}_{4}\right)$, filtered, and concentrated to dryness. The resulting crude extracts were purified by Sephadex LH-20 (GE Healthcare, Chicago, IL, USA) with methanol. Each fraction was subjected to a bioassay using strain KA20 as a test organism according to our previous report [11]. Active fractions were collected and purified by a series of silica gel column chromatography with two different solvent systems of $\mathrm{CHCl}_{3}-\mathrm{MeOH}(50: 1, v / v)$ and toluene-EtOAc $(3: 1, v / v)$. A mixture of two active components (KA54-SRB1 and KA54-SRB2) (100 $\mathrm{gg}$ from $30 \mathrm{~L}$ culture) was analyzed by ESI-MS and NMR (Figure 2). 

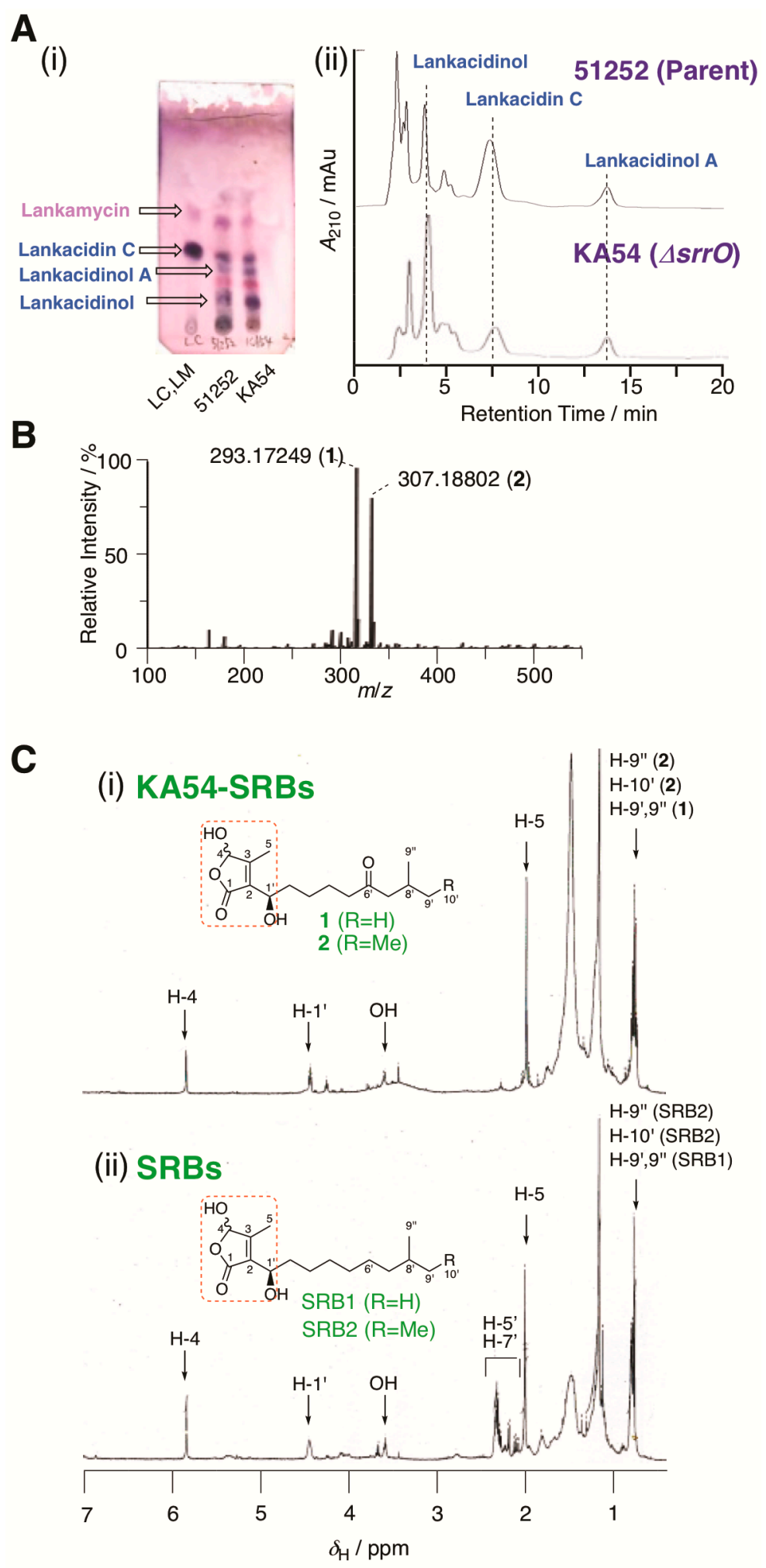

Figure 2. Metabolite analysis of strain KA54. (A) (i) TLC analysis of metabolites in strains KA54 (srrO mutant) and 51252 (parent). LC; standard sample of lankacidin C. (ii) HPLC analysis of metabolites in strains KA54 and 51252. Chromatogram was monitored by UV absorbance at $230 \mathrm{~nm}$. Structures of lankacidin C and lankamycin were displayed in Supplementary Figure S1. (B) ESI-MS spectrum of KA54-SRBs (1 and 2). (C) ${ }^{1} \mathrm{H}$ NMR spectrum of (i) KA54-SRBs (1 and 2) and (ii) SRBs.

Compound 1 (KA54-SRB1): high resolution ESI-MS; observed $m / z 293.1727\left[\mathrm{M}+\mathrm{Na}^{+}{ }^{\text {(calcd for }}\right.$ $\mathrm{C}_{15} \mathrm{H}_{26} \mathrm{O}_{4} \mathrm{Na}$, 293.1729). 
Compound 2 (KA54-SRB2): high resolution ESI-MS; observed $m / z 307.1883[\mathrm{M}+\mathrm{Na}]^{+}$(calcd for $\mathrm{C}_{16} \mathrm{H}_{28} \mathrm{O}_{4} \mathrm{Na}$, 307.1885).

Nevertheless, the low quantity of active components (100 $\mu \mathrm{g}$ from $30 \mathrm{~L}$ culture), compounds 1 and 2 were separated by repeated runs of HPLC ( $25 \%$ aqueous acetonitrile containing $0.1 \%$ trifluoroacetic acid) at $10.6 \mathrm{~min}$ and $17.1 \mathrm{~min}$, respectively. Their C-1' stereochemistry was confirmed by chiral HPLC using synthetic 6'-deoxo-SRBs (details were shown in Sections 2.7 and 3.3).

\subsection{Synthesis of $6^{\prime}$-deoxo-SRBs}

6-Hydroxyhexyl $p$-toluenesulfonate (4)

To a solution of 1,6-hexanediol (3) $(2.03 \mathrm{~g}, 17.2 \mathrm{mmol})$, triethylamine $(7.00 \mathrm{~mL}, 50.5 \mathrm{mmol})$, and 4,4-dimethylaminopyridine (50 $\mathrm{mg}, 0.41 \mathrm{mmol}$ ) in $\mathrm{CH}_{2} \mathrm{Cl}_{2}(70 \mathrm{~mL}$ ) was added $p$-toluenesulfonyl chloride $(3.28 \mathrm{~g}, 17.2 \mathrm{mmol})$ at $0{ }^{\circ} \mathrm{C}$, and the mixture was stirred at room temperature for $1 \mathrm{~h}$. Water $(50 \mathrm{~mL})$ was added, and the mixture was extracted with EtOAc twice. The combined organic phases were washed with brine, dried $\left(\mathrm{Na}_{2} \mathrm{SO}_{4}\right)$, filtered, and concentrated to dryness. The residue was purified over silica gel with hexane-EtOAc $(1: 1-1: 2, v / v)$ to give $4(2.34 \mathrm{~g}, 50 \%)$ as a colorless oil.

${ }^{1} \mathrm{H}-\mathrm{NMR}\left(\mathrm{CDCl}_{3}\right): \delta=1.33(\mathrm{~m}, 4 \mathrm{H}), 1.51(\mathrm{~m}, 2 \mathrm{H}), 1.65(\mathrm{~m}, 2 \mathrm{H}), 2.45(\mathrm{~s}, 3 \mathrm{H}), 3.60(\mathrm{t}, J=6.5 \mathrm{~Hz}$, $2 \mathrm{H}), 4.02(\mathrm{t}, J=6.3 \mathrm{~Hz}, 2 \mathrm{H}), 7.35(\mathrm{~d}, J=8.0 \mathrm{~Hz}, 2 \mathrm{H}), 7.78 \mathrm{ppm}(\mathrm{d}, J=8.0 \mathrm{~Hz}, 2 \mathrm{H}) ;{ }^{13} \mathrm{C}-\mathrm{NMR}\left(\mathrm{CDCl}_{3}\right)$ : $\delta=21.6,25.0,25.1,28.7,32.3,62.5,127.8,129.8,133.0,144.7$; High resolution ESI-MS: observed $\mathrm{m} / \mathrm{z}$ $295.0975[\mathrm{M}+\mathrm{Na}]^{+}$(calcd for $\left.\mathrm{C}_{13} \mathrm{H}_{20} \mathrm{O}_{4} \mathrm{SNa}, 295.0975\right)$; IR (neat): $v=3343,2934,2860,2361,2342,1354$, $1172 \mathrm{~cm}^{-1}$.

6-((Tetrahydro-2H-pyran-2-yl)oxy)hexyl $p$-toluenesulfonate (5)

A mixture of alcohol $4(2.30 \mathrm{~g}, 8.45 \mathrm{mmol}), 3,4$-dihydro-2H-pyrane $(1.50 \mathrm{~mL}, 16.4 \mathrm{mmol})$, and $p$-toluenesulfonic acid monohydrate $(45 \mathrm{mg})$ in $\mathrm{CH}_{2} \mathrm{Cl}_{2}(30 \mathrm{~mL})$ was stirred at room temperature for $8 \mathrm{~h}$. Saturated aqueous $\mathrm{NaHCO}_{3}(20 \mathrm{~mL})$ was added in a dropwise manner, and the mixture was extracted with EtOAc twice. The combined organic phases were washed with brine, dried $\left(\mathrm{Na}_{2} \mathrm{SO}_{4}\right)$, filtered, and concentrated to dryness. The residue was purified over silica gel with hexane-EtOAc (10:1, $v / v)$ to give $5(2.85 \mathrm{~g}, 95 \%)$ as a colorless oil.

${ }^{1} \mathrm{H}-\mathrm{NMR}\left(\mathrm{CDCl}_{3}\right): \delta=1.32-1.33(\mathrm{~m}, 4 \mathrm{H}), 1.50-1.84(\mathrm{~m}, 10 \mathrm{H}), 2.45(\mathrm{~s}, 3 \mathrm{H}), 3.32-3.72(\mathrm{~m}, 2 \mathrm{H})$, $3.47-3.87(\mathrm{~m}, 2 \mathrm{H}) 4.02(\mathrm{t}, J=6.3 \mathrm{~Hz}, 2 \mathrm{H}), 4.54(\mathrm{t}, J=3.3 \mathrm{~Hz}, 1 \mathrm{H}), 7.34(\mathrm{~d}, J=8.5 \mathrm{~Hz}, 2 \mathrm{H}), 7.78 \mathrm{ppm}$ $(\mathrm{d}, J=8.0 \mathrm{~Hz}, 2 \mathrm{H}) ;{ }^{13} \mathrm{C}-\mathrm{NMR}\left(\mathrm{CDCl}_{3}\right): \delta=19.7,21.6,25.2,25.4,25.6,28.7,29.4,30.7,62.4,67.3,70.5$, 98.9, 127.8, 129.7, 133.1, 144.6; High resolution ESI-MS: observed $m / z 379.1551[\mathrm{M}+\mathrm{Na}]^{+}$(calcd for $\left.\mathrm{C}_{18} \mathrm{H}_{28} \mathrm{O}_{5} \mathrm{SNa}, 379.1550\right)$; IR (neat): $v=2938,2862,2361,2342,1354,1175 \mathrm{~cm}^{-1}$.

2-((8-Methylnonyl)oxy)tetrahydro-2H-pyran (6)

To a suspension of magnesium $(1.00 \mathrm{~g}, 41.1 \mathrm{mmol})$ and iodine $(65 \mathrm{mg})$ in THF $(20 \mathrm{~mL})$ was added 1 -bromo-2-methylpropane $(4.50 \mathrm{~mL}, 41.7 \mathrm{mmol})$ at $0{ }^{\circ} \mathrm{C}$, and the mixture was stirred at $0{ }^{\circ} \mathrm{C}$ for $30 \mathrm{~min}$. A solution of $0.5 \mathrm{M} \mathrm{Li}_{2} \mathrm{CuCl}_{4}$ in THF $(5.00 \mathrm{~mL}, 2.50 \mathrm{mmol})$ was added to the mixture at $0{ }^{\circ} \mathrm{C}$, and then a solution of tosylate $5(3.10 \mathrm{~g}, 8.70 \mathrm{mmol})$ in THF $(10 \mathrm{~mL})$ was added dropwise to the mixture at $0{ }^{\circ} \mathrm{C}$, and the mixture was stirred at $0{ }^{\circ} \mathrm{C}$ for $1 \mathrm{~h}$. Saturated aqueous $\mathrm{NH}_{4} \mathrm{Cl}(20 \mathrm{~mL})$ was added in a dropwise manner at $0{ }^{\circ} \mathrm{C}$, and the mixture was extracted with EtOAc twice. The combined organic phases were washed with brine, dried $\left(\mathrm{Na}_{2} \mathrm{SO}_{4}\right)$, filtered, and concentrated to dryness. The residue was purified over silica gel with hexane-EtOAc $(25: 1, v / v)$ to give $6(2.10 \mathrm{~g}, 99 \%)$ as a colorless oil.

${ }^{1} \mathrm{H}-\mathrm{NMR}\left(\mathrm{CDCl}_{3}\right): \delta=0.86(\mathrm{~d}, J=7.0 \mathrm{~Hz}, 6 \mathrm{H}), 1.13-1.17(\mathrm{~m}, 2 \mathrm{H}), 1.25-1.35(\mathrm{~m}, 8 \mathrm{H}), 1.50-1.60(\mathrm{~m}$, $7 \mathrm{H}), 1.71(\mathrm{~m}, 1 \mathrm{H}), 1.83(\mathrm{~m}, 1 \mathrm{H}), 3.36-3.52(\mathrm{~m}, 2 \mathrm{H}), 3.71-3.90(\mathrm{~m}, 2 \mathrm{H}), 4.58(\mathrm{t}, J=3.5 \mathrm{~Hz}, 1 \mathrm{H}) ;{ }^{13} \mathrm{C}-\mathrm{NMR}$ $\left(\mathrm{CDCl}_{3}\right): \delta=14.0,19.6,22.6,25.5,25.9,29.2,29.7,30.7,31.6,33.5,62.2,67.6,98.8$; High resolution ESI-MS:

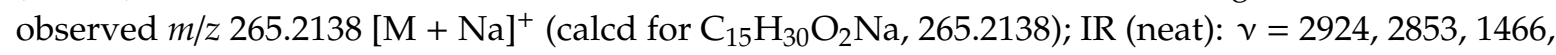
$1366,1352,1130,1117,1034,1022 \mathrm{~cm}^{-1}$.

8-Methylnonan-1-ol (7)

A solution of THP ether $6(2.43 \mathrm{~g}, 10.0 \mathrm{mmol})$ and $2 \mathrm{M}$ aqueous $\mathrm{HCl}(5.0 \mathrm{~mL})$ in THF-MeOH $(40 \mathrm{~mL}, 1: 1 \mathrm{v} / \mathrm{v})$ was stirred at room temperature for $4 \mathrm{~h}$. The mixture was concentrated in vacuo. 
The residue was purified over silica gel with hexane-EtOAc $(10: 1-2: 1, v / v)$ to give alcohol 7 (1.47 $\mathrm{g}$, $92 \%$ ) as a colorless oil.

${ }^{1} \mathrm{H}-\mathrm{NMR}\left(\mathrm{CDCl}_{3}\right): \delta=0.86(\mathrm{~d}, J=7.0 \mathrm{~Hz}, 6 \mathrm{H}), 1.13-1.17(\mathrm{~m}, 2 \mathrm{H}), 1.23-1.38(\mathrm{~m}, 8 \mathrm{H}), 1.49-1.68$ $(\mathrm{m}, 3 \mathrm{H}) 3.60(\mathrm{t}, J=6.5 \mathrm{~Hz}, 2 \mathrm{H}) ;{ }^{13} \mathrm{C}-\mathrm{NMR}\left(\mathrm{CDCl}_{3}\right): \delta=22.6,25.7,27.3,27.9,29.5,29.8,32.8,39.0,63.1$; High resolution GC-CI-MS: observed $m / z$ 157.1589 [M - H] $]^{+}$(calcd for $\left.\mathrm{C}_{10} \mathrm{H}_{21} \mathrm{O}, 157.1592\right)$; IR (neat): $v=3329,2955,2928,2859,1458,1057 \mathrm{~cm}^{-1}$.

8-Methylnonanal (8)

A mixture of alcohol $7(1.02 \mathrm{~g}, 6.44 \mathrm{mmol})$, pyridinium chlorochromate $(2.48 \mathrm{~g}, 11.5 \mathrm{mmol})$, and sodium acetate $(228 \mathrm{mg}, 2.78 \mathrm{mmol})$ in $\mathrm{CH}_{2} \mathrm{Cl}_{2}(50 \mathrm{~mL})$ was stirred at room temperature for $2 \mathrm{~h}$. The mixture was diluted with ether $(70 \mathrm{~mL})$, and filtered through a pad of Celite. The filtrate and washings were concentrated in vacuo. The residue was purified over silica gel with hexane-EtOAc $(15: 1, v / v)$ to give aldehyde $8(869 \mathrm{mg}, 86 \%)$ as a colorless oil.

${ }^{1} \mathrm{H}-\mathrm{NMR}\left(\mathrm{CDCl}_{3}\right): \delta=0.86(\mathrm{~d}, J=7.0 \mathrm{~Hz}, 6 \mathrm{H}), 1.16(\mathrm{~m}, 2 \mathrm{H}), 1.26-1.33(\mathrm{~m}, 6 \mathrm{H}), 1.51(\mathrm{~m}, 1 \mathrm{H})$, $1.63(\mathrm{~m}, 2 \mathrm{H}), 2.42(\mathrm{dt}, J=1.5$ and $7.3 \mathrm{~Hz}, 2 \mathrm{H}), 9.76(\mathrm{t}, J=2.0 \mathrm{~Hz}, 1 \mathrm{H}) ;{ }^{13} \mathrm{C}-\mathrm{NMR}\left(\mathrm{CDCl}_{3}\right): \delta=22.1$, 22.6, 27.1, 27.9, 29.2, 29.6, 38.9, 43.9, 203.0; High resolution GC-CI-MS: observed m/z 157.1589 [M + H] ${ }^{+}$ (calcd for $\left.\mathrm{C}_{10} \mathrm{H}_{21} \mathrm{O}, 157.1592\right)$; IR (neat): $v=2951,2924,2855,1726,1709,1466 \mathrm{~cm}^{-1}$.

$\left(1^{\prime} R\right)-2-\left(1^{\prime}-H y d r o x y l-8\right.$-methylnonyl)-3-methyl-4-(L-menthyloxy)but-2-en-1,4-olide (10a) and (1'S)-2-(1'-Hydroxyl-8'-methylnonyl)-3-methyl-4-(L-menthyloxy)but-2-en-1,4-olide (10b).

A solution of $n$-butyl lithium $(2.10 \mathrm{~mL}, 1.64 \mathrm{M}$ in hexane, $3.44 \mathrm{mmol})$ was added in a dropwise manner to a solution of diisopropylamine $(470 \mu \mathrm{L}, 3.34 \mathrm{mmol})$ in THF $(7 \mathrm{~mL})$ at $-78^{\circ} \mathrm{C}$. After $30 \mathrm{~min}$ of stirring, hexamethylphosphoric triamide (HMPA) $(2.00 \mathrm{~mL})$ was added to the mixture at $-78{ }^{\circ} \mathrm{C}$. A solution of 3-methyl-4-(L-menthyloxy)but-2-en-1,4-olide (9) (745 mg, 2.95 mmol) [11,35,36] in THF $(7 \mathrm{~mL})$ was added to the mixture at $-78{ }^{\circ} \mathrm{C}$ over $15 \mathrm{~min}$, and the mixture was further stirred at the same temperature for $1 \mathrm{~h}$. Then, a solution of aldehyde 8 (685 mg, $4.38 \mathrm{mmol})$ in THF (7 mL) was added dropwise at $-78{ }^{\circ} \mathrm{C}$ over $10 \mathrm{~min}$, and the mixture was further stirred at the same temperature for $1.5 \mathrm{~h}$. Saturated aqueous $\mathrm{NH}_{4} \mathrm{Cl}(20 \mathrm{~mL})$ was added to the mixture, and the mixture was extracted with $\mathrm{CH}_{2} \mathrm{Cl}_{2}$ twice. The combined organic phases were washed with brine, dried $\left(\mathrm{Na}_{2} \mathrm{SO}_{4}\right)$, filtered, and concentrated to dryness. The residue was purified by silica gel chromatography with hexane-EtOAc $(10: 1-7: 1, v / v)$ to give a $2: 1$ mixture of $\mathbf{1 0 a}$ and $\mathbf{1 0 b}(452 \mathrm{mg}, 38 \%)$ as a colorless oil, which were further separated by repeated runs of flash chromatography with hexane-EtOAc $(10: 1, v / v)$. Their absolute configuration at C-1' was established by the modified Mosher method [37].

Compound 10a: $[\alpha]_{\mathrm{D}}{ }^{20}=-83.1\left(c=0.32, \mathrm{CHCl}_{3}\right) ;{ }^{1} \mathrm{H}-\mathrm{NMR}\left(\mathrm{CDCl}_{3}\right): \delta=0.86(\mathrm{~d}, \mathrm{~J}=6.4 \mathrm{~Hz}, 6 \mathrm{H})$, 1.15 (m, 2H), 1.26-1.33 (m, 6H), 1.41-1.55 (m, 2H), $1.65(\mathrm{~m}, 2 \mathrm{H}), 1.84(\mathrm{~m}, 1 \mathrm{H}), 1.98(\mathrm{~s}, 3 \mathrm{H}), 2.88(\mathrm{br}, 1 \mathrm{H})$, $4.46(\mathrm{br}, J=7.5 \mathrm{~Hz}, 1 \mathrm{H}), 5.70(\mathrm{~s}, 1 \mathrm{H})$, menthyl resonances: $0.81(\mathrm{~d}, J=6.8 \mathrm{~Hz}, 3 \mathrm{H}), 0.86(\mathrm{~m}, 1 \mathrm{H}), 0.88(\mathrm{~d}$, $J=7.1 \mathrm{~Hz}, 3 \mathrm{H}), 0.96(\mathrm{~d}, J=6.4 \mathrm{~Hz}, 3 \mathrm{H}), 1.02(\mathrm{~m}, 2 \mathrm{H}), 1.22-1.27(\mathrm{~m}, 1 \mathrm{H}), 1.28-1.42(\mathrm{~m}, 1 \mathrm{H}), 1.64-1.70(\mathrm{~m}$, $2 \mathrm{H}), 2.08-2.14(\mathrm{~m}, 2 \mathrm{H}), 3.62(\mathrm{dt}, J=4.3$ and $11 \mathrm{~Hz}, 1 \mathrm{H}) ;{ }^{13} \mathrm{C}-\mathrm{NMR}\left(\mathrm{CDCl}_{3}\right): \delta=11.5,22.6,25.5,27.2,27.9$, 29.3, 29.7, 36.7, 38.9, 67.0, 100.8, 130.6, 155.3, 171.4, menthyl resonances: 15.9, 20.8, 22.2, 23.2, 25.3, 31.4, 34.2, 40.5, 47.7, 79.5; High resolution ESI-MS: observed $m / z 409.3320[\mathrm{M}+\mathrm{H}]^{+}$(calcd for $\mathrm{C}_{25} \mathrm{H}_{45} \mathrm{O}_{4}$, 409.3312); IR (neat): $v=2951,2922,2868,2854,1751,1456,1384,1367,1331,1126,1093,945 \mathrm{~cm}^{-1}$.

Compound 10b: $[\alpha]_{\mathrm{D}}{ }^{28}=-62.5\left(c=0.460, \mathrm{CHCl}_{3}\right) ;{ }^{1} \mathrm{H}-\mathrm{NMR}\left(\mathrm{CDCl}_{3}\right): \delta=0.86(\mathrm{~d}, J=6.7 \mathrm{~Hz}, 6 \mathrm{H})$, $1.15(\mathrm{~m}, 2 \mathrm{H}), 1.24-1.33(\mathrm{~m}, 6 \mathrm{H}), 1.40-1.55(\mathrm{~m}, 2 \mathrm{H}), 1.66(\mathrm{~m}, 2 \mathrm{H}), 1.83(\mathrm{~m}, 1 \mathrm{H}), 1.99(\mathrm{~s}, 3 \mathrm{H}), 2.77(\mathrm{br}, 1 \mathrm{H})$, $4.47(\mathrm{br}, J=6.4 \mathrm{~Hz}, 1 \mathrm{H}), 5.71(\mathrm{~s}, 1 \mathrm{H})$, menthyl resonances: $0.81(\mathrm{~d}, J=6.8 \mathrm{~Hz}, 3 \mathrm{H}), 0.86(\mathrm{~m}, 1 \mathrm{H}), 0.88(\mathrm{~d}$, $J=7.1 \mathrm{~Hz}, 3 \mathrm{H}), 0.96(\mathrm{~d}, J=6.4 \mathrm{~Hz}, 3 \mathrm{H}), 1.02(\mathrm{~m}, 2 \mathrm{H}), 1.22-1.27(\mathrm{~m}, 1 \mathrm{H}), 1.28-1.42(\mathrm{~m}, 1 \mathrm{H}), 1.64-1.70(\mathrm{~m}$, $2 \mathrm{H}), 2.08-2.14(\mathrm{~m}, 2 \mathrm{H}), 3.62(\mathrm{dt}, J=4.3$ and $11 \mathrm{~Hz}, 1 \mathrm{H}) ;{ }^{13} \mathrm{C}-\mathrm{NMR}\left(\mathrm{CDCl}_{3}\right): \delta=11.5,22.6,25.5,27.2,27.9$, 29.3, 29.7, 36.7, 38.9, 67.0, 100.8, 130.6, 155.3, 171.4, menthyl resonances: 15.9, 20.8, 22.2, 23.2, 25.3, 31.4, 34.2, 40.5, 47.7, 79.5; High resolution ESI-MS: observed $m / z 409.3315[\mathrm{M}+\mathrm{H}]^{+}$(calcd for $\mathrm{C}_{25} \mathrm{H}_{45} \mathrm{O}_{4}$ ， 409.3312); IR (neat): $v=2951,2922,2868,2855,1751,1456,1385,1368,1330,1094,945 \mathrm{~cm}^{-1}$.

6'-Deoxo-SRB1a (1a)

To a solution of menthyl ester 10a $(34 \mathrm{mg}, 83.2 \mu \mathrm{mol})$ in $\mathrm{CH}_{2} \mathrm{Cl}_{2}(4.0 \mathrm{~mL})$ was added $10 \% \mathrm{BBr}_{3}$ solution in $\mathrm{CH}_{2} \mathrm{Cl}_{2}(400 \mu \mathrm{L}, 420 \mu \mathrm{mol})$ at $-78^{\circ} \mathrm{C}$, and the mixture was stirred at $-78^{\circ} \mathrm{C}$ for $3 \mathrm{~h}$. Saturated 
aqueous $\mathrm{NaHCO}_{3}(3 \mathrm{~mL})$ was carefully added, and the mixture was extracted with EtOAc twice. The combined organic phases were washed with brine, dried $\left(\mathrm{Na}_{2} \mathrm{SO}_{4}\right)$, filtered, and concentrated to dryness. The residue was purified by silica gel chromatography with hexane-EtOAc $(2: 1, v / v)$ to give $6^{\prime}$-deoxo-SRB1a (1a) (17 mg, 79\%) as a colorless oil.

Mixture of C-4 epimers: $[\alpha]_{\mathrm{D}}{ }^{18}=+126.4\left(c=0.11, \mathrm{CHCl}_{3}\right) ;{ }^{1} \mathrm{H}-\mathrm{NMR}\left(\mathrm{CDCl}_{3}\right): \delta=0.86(\mathrm{~d}$, $J=6.7 \mathrm{~Hz}, \mathrm{H}-9^{\prime}$ and H-9', $\left.6 \mathrm{H}\right), 1.15$ (m, H-7', 2H), 1.26 (m, H-3'a, H-4', and H-6', 5H), 1.29 (m, H-5', 2H), 1.39 (m, H-3'b, 1H), 1.51 (m, H-8', 1H), 1.65 (m, H-2'a, 1H), 1.81 (m, H-2'b, 1H), 2.07 (s, H-5, $3 \mathrm{H}), 4.47\left(\mathrm{~m}, \mathrm{H}-1^{\prime}, 1 \mathrm{H}\right), 5.85$ (brs, $\left.\mathrm{H}-4,1 \mathrm{H}\right) ;{ }^{13} \mathrm{C}-\mathrm{NMR}\left(\mathrm{CDCl}_{3}\right): \delta=11.4 / 11.6(\mathrm{C}-5), 22.6\left(\mathrm{C}-9^{\prime}\right.$ and C-9"), 25.5 (C-3'), 27.3 (C-6'), 27.9 (C-8'), 29.3 (C-5'), 29.7/29.8 (C-4'), 36.0/36.2 (C-2'), 39.0 (C-7'), 66.7 (C-1'), 98.8 (C-4), 130.1/130.5 (C-2), 157.9 (C-3), 171.7/172.0 (C-1); High resolution ESI-MS: observed m/z $293.1718[\mathrm{M}+\mathrm{Na}]^{+}$(calcd for $\left.\mathrm{C}_{15} \mathrm{H}_{26} \mathrm{O}_{4} \mathrm{Na}, 293.1723\right) ; \mathrm{IR}$ (neat): $v=3399,2957,2924,2855,2361,2342$, $1749,1734 \mathrm{~cm}^{-1}$.

\section{$6^{\prime}$-Deoxo-SRB1b (1b)}

The compound $10 \mathrm{~b}(20 \mathrm{mg}, 49 \mu \mathrm{mol})$ was treated in the same manner as described for the preparation of 1a to give $6^{\prime}$-deoxo-SRB1b (1b) $(8.1 \mathrm{mg}, 62 \%)$ as a colorless oil.

Mixture of C-4 epimers: $[\alpha]_{\mathrm{D}}{ }^{25}=+1.32\left(c=0.180, \mathrm{CHCl}_{3}\right) ;{ }^{1} \mathrm{H}-\mathrm{NMR}\left(\mathrm{CDCl}_{3}\right): \delta=0.86(\mathrm{~d}$, $J=6.7 \mathrm{~Hz}, \mathrm{H}-9^{\prime}$ and H-9", $\left.6 \mathrm{H}\right), 1.15$ (m, H-7', 2H), 1.26 (m, H-3'a, H-4', and H-6', 5H), 1.29 (m, H-5', 2H), 1.39 (m, H-3'b, 1H), 1.51 (m, H-8' $1 \mathrm{H}), 1.65$ (m, H-2'a, 1H), 1.81 (m, H-2'b, 1H), 2.07 (s, H-5, 3H), $4.47\left(\mathrm{~m}, \mathrm{H}-1^{\prime}, 1 \mathrm{H}\right), 5.85$ (brs, H-4, 1H); ${ }^{13} \mathrm{C}-\mathrm{NMR}\left(\mathrm{CDCl}_{3}\right): \delta=11.4 / 11.6(\mathrm{C}-5), 22.6\left(\mathrm{C}-9^{\prime}\right.$ and $\left.\mathrm{C}-9^{\prime \prime}\right), 25.5$ (C-3'), $27.3\left(\mathrm{C}-6^{\prime}\right), 27.9\left(\mathrm{C}-8^{\prime}\right), 29.3\left(\mathrm{C}-5^{\prime}\right), 29.8\left(\mathrm{C}-4^{\prime}\right), 36.0 / 36.4\left(\mathrm{C}-2^{\prime}\right), 39.0\left(\mathrm{C}-7^{\prime}\right), 66.8\left(\mathrm{C}-1^{\prime}\right)$, 98.7/98.8 (C-4), 130.1/130.6 (C-2), 157.4 (C-3), 171.6/171.7 (C-1); High resolution ESI-MS: observed m/z 293.1720 $[\mathrm{M}+\mathrm{Na}]^{+}$(calcd for $\mathrm{C}_{15} \mathrm{H}_{26} \mathrm{O}_{4} \mathrm{Na}, 293.1723$ ); IR (neat): $v=3361,2951,2924,2855,1743,1466 \mathrm{~cm}^{-1}$.

2-(((S)-8-Methyldecyl)oxy)tetrahydro-2H-pyran (11)

A suspension of $(S)-(+)-1$-chloro-2-methylbutane $(5.00 \mathrm{~mL}, 41.7 \mathrm{mmol})$, magnesium $(1.01 \mathrm{~g}$, $41.7 \mathrm{mmol})$, and iodine $(50 \mathrm{mg})$ in THF $(20 \mathrm{~mL})$ was refluxed for $45 \mathrm{~min}$. A solution of $0.5 \mathrm{M} \mathrm{Li}_{2} \mathrm{CuCl}_{4}$ solution in THF $(5.00 \mathrm{~mL}, 2.50 \mathrm{mmol})$ was added to the mixture at $0{ }^{\circ} \mathrm{C}$, and then a solution of tosylate $5(2.31 \mathrm{~g}, 6.48 \mathrm{mmol})$ in THF $(9 \mathrm{~mL})$ was added dropwise to the mixture at $0{ }^{\circ} \mathrm{C}$, and the mixture was stirred at $0{ }^{\circ} \mathrm{C}$ for $1 \mathrm{~h}$. Saturated aqueous $\mathrm{NH}_{4} \mathrm{Cl}(20 \mathrm{~mL})$ was added in a dropwise manner at $0{ }^{\circ} \mathrm{C}$, and the mixture was extracted with EtOAc twice. The combined organic phases were washed with brine, dried $\left(\mathrm{Na}_{2} \mathrm{SO}_{4}\right)$, filtered, and concentrated to dryness. The residue was purified over silica gel with hexane-EtOAc $(25: 1, v / v)$ to give THP-ether $11(1.65 \mathrm{~g}, 99 \%)$ as a colorless oil.

Diastereomer mixture: ${ }^{1} \mathrm{H}-\mathrm{NMR}\left(\mathrm{CDCl}_{3}\right): \delta=0.83(\mathrm{~d}, J=6.4 \mathrm{~Hz}, 3 \mathrm{H}), 0.85(\mathrm{t}, J=6.7 \mathrm{~Hz}, 3 \mathrm{H}), 1.12$ $(\mathrm{m}, 2 \mathrm{H}), 1.24-1.36(\mathrm{~m}, 12 \mathrm{H}), 1.53-1.61(\mathrm{~m}, 5 \mathrm{H}), 1.72(\mathrm{~m}, 1 \mathrm{H}), 1.83(\mathrm{~m}, 1 \mathrm{H}), 3.36-3.52(\mathrm{~m}, 2 \mathrm{H}), 3.71-3.90$ $(\mathrm{m}, 2 \mathrm{H}), 4.58(\mathrm{t}, J=3.5 \mathrm{~Hz}, 1 \mathrm{H}), 5.70(\mathrm{~s}, 1 \mathrm{H}) ;{ }^{13} \mathrm{C}-\mathrm{NMR}\left(\mathrm{CDCl}_{3}\right): \delta=11.4,19.2,19.7,25.5,26.2,27.0,29.5$, 29.5, 29.8, 29.9, 30.8, 34.4, 36.6, 62.3, 67.7, 98.8; High resolution ESI-MS: observed $m / z 279.2295$ [M + Na] ${ }^{+}$ (calcd for $\left.\mathrm{C}_{16} \mathrm{H}_{32} \mathrm{O}_{2} \mathrm{Na}, 279.2295\right) ; \mathrm{IR}$ (neat): $v=2955,2924,2852,1458,1377,1034,1022 \mathrm{~cm}^{-1}$.

(S)-8-Methyldecan-1-ol (12)

The THP-ether 11 (1.65 g, $6.43 \mathrm{mmol})$ was treated in the same manner as described for the preparation of 7 to give alcohol $12(1.10 \mathrm{~g}, 99 \%)$ as a colorless oil.

$[\alpha]_{D}{ }^{26}=+3.50\left(c=1.00, \mathrm{CHCl}_{3}\right) ;{ }^{1} \mathrm{H}-\mathrm{NMR}\left(\mathrm{CDCl}_{3}\right): \delta=0.84(\mathrm{~d}, J=6.1 \mathrm{~Hz}, 3 \mathrm{H}), 0.85(\mathrm{t}, J=7.3 \mathrm{~Hz}$, $3 \mathrm{H}), 1.07-1.16(\mathrm{~m}, 2 \mathrm{H}), 1.26-1.36(\mathrm{~m}, 11 \mathrm{H}), 1.56(\mathrm{~m}, 2 \mathrm{H}), 3.62(\mathrm{t}, \mathrm{J}=6.7,2 \mathrm{H}) ;{ }^{13} \mathrm{C}-\mathrm{NMR}\left(\mathrm{CDCl}_{3}\right): \delta=11.3$, 19.1, 25.7, 27.0, 29.4, 29.9, 32.7, 34.3, 36.6, 62.9; High resolution GC-CI-MS: observed $\mathrm{m} / \mathrm{z} 171.1744$ $[\mathrm{M}+\mathrm{Na}]^{+}$(calcd for $\mathrm{C}_{11} \mathrm{H}_{23} \mathrm{O}, 171.1749$ ); IR (neat): $v=3318,2959,2924,2853,1458,1377,1055 \mathrm{~cm}^{-1}$.

(S)-8-Methyldecanal (13)

The compound 12 ( $810 \mathrm{mg}, 4.70 \mathrm{mmol})$ was treated in the same manner as described for the preparation of $\mathbf{8}$ to give $\mathbf{1 3}(746 \mathrm{mg}, 93 \%)$ as a colorless oil.

$[\alpha]_{\mathrm{D}}^{27}=+0.75\left(c=1.00, \mathrm{CHCl}_{3}\right) ;{ }^{1} \mathrm{H}-\mathrm{NMR}\left(\mathrm{CDCl}_{3}\right): \delta=0.84(\mathrm{~d}, J=6.5 \mathrm{~Hz}, 3 \mathrm{H}), 0.85(\mathrm{t}, J=7.0 \mathrm{~Hz}$, $3 \mathrm{H}), 1.07-1.16(\mathrm{~m}, 2 \mathrm{H}), 1.24-1.30(\mathrm{~m}, 9 \mathrm{H}), 1.62(\mathrm{~m}, 2 \mathrm{H}), 2.42(\mathrm{dt}, J=2.4$ and $7.3,2 \mathrm{H}), 9.77(\mathrm{t}, J=2.4 \mathrm{~Hz}$, 1H); ${ }^{13} \mathrm{C}-\mathrm{NMR}\left(\mathrm{CDCl}_{3}\right): \delta=11.4,19.2,22.1,26.9,29.2,29.5,29.7,34.4,36.5,43.9,203.0$; High resolution 
GC-CI-MS: observed $m / z$ 171.1751 [M + H] ${ }^{+}$(calcd for $\mathrm{C}_{10} \mathrm{H}_{23} \mathrm{O}, 171.1749$ ); IR (neat): $v=2957,2924$, $2855,1707,1458,1412,1287 \mathrm{~cm}^{-1}$.

$\left(1^{\prime} R, 8 S\right)-2-\left(1^{\prime}\right.$-Hydroxyl-8'-methyldecyl)-3-methyl-4-(L-menthyloxy)but-2-en-1,4-olide (14a) and (1'S,8S)-2-(1'-Hydroxyl-8'-methyldecyl)-3-methyl-4-(L-menthyloxy)but-2-en-1,4-olide (14b).

Compound 13 (696 mg, $4.09 \mathrm{mmol}$ ) and L-menthyloxy-butenolide $(962 \mathrm{mg}, 3.81 \mathrm{mmol})$ were treated in the same manner as described for the preparation of $\mathbf{1 0}$ to give a 2:1 mixture of 14a and $\mathbf{1 4 b}$ (806 $\mathrm{mg}, 50 \%$ ) as a colorless oil, which was also further separated by flash chromatography.

Compound 14a: $[\alpha]_{\mathrm{D}}{ }^{23}=-70.7\left(c=1.71, \mathrm{CHCl}_{3}\right) ;{ }^{1} \mathrm{H}-\mathrm{NMR}\left(\mathrm{CDCl}_{3}\right): \delta=0.84(\mathrm{~d}, J=6.4 \mathrm{~Hz}, 3 \mathrm{H})$, $0.85(\mathrm{~d}, J=7.0 \mathrm{~Hz}, 3 \mathrm{H}), 1.24-1.34(\mathrm{~m}, 10 \mathrm{H}), 1.63-1.66(\mathrm{~m}, 2 \mathrm{H}), 1.84(\mathrm{~m}, 1 \mathrm{H}), 1.98(\mathrm{~s}, 3 \mathrm{H}), 2.83(\mathrm{br}, 1 \mathrm{H})$, $4.45(\mathrm{br}, J=7.5 \mathrm{~Hz}, 1 \mathrm{H}), 5.70(\mathrm{~s}, 1 \mathrm{H})$, menthyl resonances: $0.81(\mathrm{~d}, J=6.8 \mathrm{~Hz}, 3 \mathrm{H}), 0.86(\mathrm{~m}, 1 \mathrm{H}), 0.88(\mathrm{~d}$, $J=7.1 \mathrm{~Hz}, 3 \mathrm{H}), 0.96(\mathrm{~d}, J=6.4 \mathrm{~Hz}, 3 \mathrm{H}), 1.02(\mathrm{~m}, 2 \mathrm{H}), 1.22-1.27(\mathrm{~m}, 1 \mathrm{H}), 1.28-1.42(\mathrm{~m}, 1 \mathrm{H}), 1.64-1.70(\mathrm{~m}$, $2 \mathrm{H}), 2.08-2.14(\mathrm{~m}, 2 \mathrm{H}), 3.62(\mathrm{dt}, J=4.3$ and $11 \mathrm{~Hz}, 1 \mathrm{H}) ;{ }^{13} \mathrm{C}-\mathrm{NMR}\left(\mathrm{CDCl}_{3}\right): \delta=11.4,11.5,19.2,25.5$, 27.0, 29.4, 29.5, 29.9, 34.3, 36.6, 36.7, 67.0, 100.9, 130.6, 155.3, 171.4, menthyl resonances: 15.9, 20.8, 22.2, 23.2, 25.4, 31.5, 34.2, 40.5, 47.7, 79.5; High resolution ESI-MS: observed $m / z 445.3289[\mathrm{M}+\mathrm{Na}]^{+}$(calcd for $\mathrm{C}_{26} \mathrm{H}_{46} \mathrm{O}_{4} \mathrm{Na}, 445.3288$ ); IR (neat): $v=2953,2922,2868,2854,1751,1456,1331,1096,943 \mathrm{~cm}^{-1}$.

Compound 14b: $[\alpha]_{\mathrm{D}}{ }^{22}=-95.4\left(c=1.00, \mathrm{CHCl}_{3}\right) \cdot{ }^{1} \mathrm{H}-\mathrm{NMR}\left(\mathrm{CDCl}_{3}\right):{ }^{1} \mathrm{H}-\mathrm{NMR}\left(\mathrm{CDCl}_{3}\right): \delta=0.84$ $(\mathrm{d}, J=6.4,3 \mathrm{H}), 0.85(\mathrm{~d}, J=7.0,3 \mathrm{H}), 1.24-1.34(\mathrm{~m}, 10 \mathrm{H}), 1.63(\mathrm{~m}, 2 \mathrm{H}), 1.83(\mathrm{~m}, 1 \mathrm{H}), 1.98(\mathrm{~s}, 3 \mathrm{H}), 2.77$ $(\mathrm{br}, 1 \mathrm{H}), 4.47(\mathrm{br}, 1 \mathrm{H}), 5.71(\mathrm{~s}, 1 \mathrm{H})$, menthyl resonances: $0.81(\mathrm{~d}, J=6.8 \mathrm{~Hz}, 3 \mathrm{H}), 0.86(\mathrm{~m}, 1 \mathrm{H}), 0.88(\mathrm{~d}$, $J=7.1 \mathrm{~Hz}, 3 \mathrm{H}), 0.96(\mathrm{~d}, J=6.4 \mathrm{~Hz}, 3 \mathrm{H}), 1.02(\mathrm{~m}, 2 \mathrm{H}), 1.22-1.27(\mathrm{~m}, 1 \mathrm{H}), 1.28-1.42(\mathrm{~m}, 1 \mathrm{H}), 1.64-1.70(\mathrm{~m}$, $2 \mathrm{H}), 2.08-2.14(\mathrm{~m}, 2 \mathrm{H}), 3.62(\mathrm{dt}, J=4.3$ and $11 \mathrm{~Hz}, 1 \mathrm{H}) ;{ }^{13} \mathrm{C}-\mathrm{NMR}\left(\mathrm{CDCl}_{3}\right): \delta=11.4,11.5,19.2,25.6$, 27.0, 29.4, 29.5, 29.9, 34.3, 36.5, 66.9, 100.7, 130.7, 155.3, 171.4, menthyl resonances: 15.7, 20.9, 22.2, 23.1, 25.2, 31.4, 34.2, 40.4, 47.7, 79.5; High resolution ESI-MS: observed $m / z 445.3287$ [M + Na] ${ }^{+}$(calcd for $\mathrm{C}_{26} \mathrm{H}_{46} \mathrm{O}_{4} \mathrm{Na}$, 445.3288); IR (neat): $v=2953,2922,2868,2855,1751,1456,1369,1331,1096,943 \mathrm{~cm}^{-1}$.

6'-Deoxo-SRB2a (2a)

The compound 14a (19 $\mathrm{mg}, 45 \mu \mathrm{mol})$ was treated in the same manner as described for the preparation of 1a to give $6^{\prime}$-deoxo-SRB2a (2a) $(10 \mathrm{mg}, 80 \%)$ as a colorless oil.

Mixture of C-4 epimers: $[\alpha]_{\mathrm{D}}{ }^{20}=+78.3\left(c=0.180, \mathrm{CHCl}_{3}\right) ;{ }^{1} \mathrm{H}-\mathrm{NMR}\left(\mathrm{CDCl}_{3}\right): \delta=0.83(\mathrm{~d}, J=6.4 \mathrm{~Hz}$, H-9", 3H), 0.86 (t, J = 6.7 Hz, H-10', 3H), 1.07 (m, H-7'a, 1H), 1.12 (m, H-9'a, 1H), 1.28 (m, H-3'a, H-4', H-5', H-6' , H-7'b, H-8', and H-9'b, 10H), 1.40 (m, H-3'b, 1H), 1.66 (m, H-2'a, 1H), 1.81 (br, H-2'b, 1H), $2.07(\mathrm{~s}, \mathrm{H}-5,3 \mathrm{H}), 4.46\left(\mathrm{~m}, \mathrm{H}-1^{\prime}, 1 \mathrm{H}\right), 5.85(\mathrm{brs}, \mathrm{H}-4,1 \mathrm{H}) ;{ }^{13} \mathrm{C}-\mathrm{NMR}\left(\mathrm{CDCl}_{3}\right): \delta=11.4\left(\mathrm{C}-10^{\prime}\right), 11.4 / 11.5$ (C-5), 19.2 (C-9"), 25.5 (C-3'), 27.0 (C-6'), 29.4 (C-5'), 29.5 (C-9'), 29.9 (C-4'), 34.4 (C-8'), 36.2 (C-2'), 36.6 (C-7'), 66.8 (C-1'), 98.6/98.7 (C-4), 130.2 (C-2), 157.2 (C-3), 171.5 (C-1); High resolution ESI-MS: observed $m / z 307.1881[\mathrm{M}+\mathrm{Na}]^{+}$(calcd for $\left.\mathrm{C}_{16} \mathrm{H}_{28} \mathrm{O}_{4} \mathrm{Na}, 307.1880\right)$; IR (neat): $v=3385,2955,2924$, $2855,1736 \mathrm{~cm}^{-1}$.

$6^{\prime}$-Deoxo-SRB2b (2b)

The compound 14b (15 mg, $36 \mu \mathrm{mol})$ was treated in the same manner as described for the preparation of $\mathbf{1 a}$ to give $6^{\prime}$-deoxo-SRB2b $(\mathbf{2 b})(7.3 \mathrm{mg}, 72 \%)$ as a colorless oil.

Mixture of C-4 epimers: $[\alpha]_{\mathrm{D}}{ }^{20}=-5.09\left(c=0.530, \mathrm{CHCl}_{3}\right) ;{ }^{1} \mathrm{H}-\mathrm{NMR}\left(\mathrm{CDCl}_{3}\right): \delta=0.83(\mathrm{~d}, J=6.4 \mathrm{~Hz}$, H-9", 3H), 0.86 (t, J = 6.7 Hz, H-10', 3H), 1.07 (m, H-7'a, 1H), 1.12 (m, H-9'a, 1H), 1.28 (m, H-3'a, H-4', H-5', H-6', H-7'b, H-8', and H-9'b, 10H), 1.40 (m, H-3'b, 1H), 1.66 (m, H-2'a, 1H), 1.81 (br, H-2'b, 1H), 2.07 (s, H-5, 3H), 4.46 (m, H-1', 1H), 5.85 (brs, H-4, 1H); ${ }^{13} \mathrm{C}-\mathrm{NMR}\left(\mathrm{CDCl}_{3}\right): \delta=11.4\left(\mathrm{C}-10^{\prime}\right), 11.4 / 11.5$ (C-5), 19.2 (C-9') , 25.5 (C-3'), 27.0 (C-6'), $29.3\left(\mathrm{C}-5^{\prime}\right), 29.5$ (C-9'), 29.8 (C-4'), 34.3 (C-8'), 36.1/36.4 (C-2'), 36.6 (C-7'), 66.8 (C-1'), 98.5/98.7 (C-4), 130.2/130.7 (C-2), 157.1 (C-3), 171.5 (C-1); High resolution ESI-MS: observed $m / z 307.1880[\mathrm{M}+\mathrm{Na}]^{+}$(calcd for $\mathrm{C}_{16} \mathrm{H}_{28} \mathrm{O}_{4} \mathrm{Na}, 307.1880$ ); IR (neat): $v=3385,2955,2924$, $2854,1736,1458 \mathrm{~cm}^{-1}$.

\subsection{Chiral HPLC Analysis}

Natural KA54-SRB1 and KA54-SRB2 were analyzed by chiral HPLC using a Chiral MB-S column (4.6 I.D. $\times 250 \mathrm{~mm}$, macroporous silica gel coated with optically active $\mathrm{N}$-substituted polymaleimides; Tokyo Kasei, Co. Ltd., Tokyo, Japan). The mobile phase was composed of two solvents; $20 \%$ aqueous 
acetonitrile with $0.1 \%$ trifluoroacetic acid (solvent $\mathrm{A}$ ) and $10 \%$ aqueous acetonitrile with $0.1 \%$ trifluoroacetic acid (solvent $\mathrm{B}$ ), and samples were eluted at a flow rate of $1.0 \mathrm{~mL} / \mathrm{min}$ with detection at $210 \mathrm{~nm}$. The linear-gradient elution program was set as follows: $100 \% \mathrm{~A}(0-10 \mathrm{~min}), 0-100 \% \mathrm{~A}$ (10-25 $\mathrm{min}), 0-100 \% \mathrm{~A}(25-35 \mathrm{~min})$ and 100\% A (35-60 min). The injection volume of each sample was $10 \mu \mathrm{L}$. Natural $6^{\prime}$-deoxo-SRB1 (1) and 6'-deoxo-SRB2 (2) were eluted at 21.1 and $53.3 \mathrm{~min}$, respectively. Synthetic $\mathbf{1 a}, \mathbf{1} \mathbf{b}, \mathbf{2 a}$, and $\mathbf{2 b}$ were eluted at 21.1, 20.2, 53.3, and $50.0 \mathrm{~min}$, respectively.

\subsection{Gel Shift Assay}

Preparation of an SrrA protein (SRB receptor) and a DNA probe containing the promoter region of $\operatorname{srr} Y(\operatorname{srr} Y p)$, a target gene of SrrA, was described previously [22,38]. The reaction mixture contained the binding buffer $(20 \mathrm{mM}$ Tris- $\mathrm{HCl}$ [pH 8.0], $100 \mathrm{mM} \mathrm{NaCl}, 1 \mathrm{mM}$ dithiothreitol, $0.1 \mathrm{mg}$ of bovine serum albumin and 5\% glycerol), $0.35 \mathrm{nM}$ labeled DNA, and $2 \mu \mathrm{M}$ SrrA protein. To analyze the ligand affinity of $6^{\prime}$-deoxo-SRB on the binding of SrrA, various concentrations of synthetic $6^{\prime}$-deoxo-SRBs and SRBs were added to the reaction mixture.

\subsection{Preparation of the Streptomyces Lividans Recombinant for SrrO Protein}

A 1.2-kb PCR fragment containing srrO (nt 145,325-144,081 complement of pSLA2-L) was amplified using the template cosmid C7 [17] and two primers, NT-srrO-OE-F and NT-srrO-OE-R (Table S1). The PCR fragment was digested with NdeI and HinDIII and cloned into pKAR3063H [23], a (His) -tag $^{-}$ containing derivative of a constitutive expression vector pHSA81 (Michihiko Kobayashi and Yoshiteru Hashimoto, personal communication), to give pNTT01 (Figure S3). The Streptomyces lividans TK64 recombinant harboring pNTT01 was grown at $28{ }^{\circ} \mathrm{C}$ for $72 \mathrm{~h}$ in YEME liquid medium ( $34 \%$ sucrose) containing $10 \mu \mathrm{g} / \mathrm{mL}$ of thiostrepton.

\subsection{Bioconversion of 6'-deoxo-SRB1 in the SrrO Recombinant}

To a 2-day-growth culture $(100 \mathrm{~mL})$ of S. lividans TK64/pNTT01 (+SrrO) was added $2 \mu \mathrm{mol}$ of the substrate, and the fed cultures were further incubated for $0-5 \mathrm{~h}$ periods. The culture supernatant was extracted with EtOAc twice, and the combined organic phase was dried $\left(\mathrm{Na}_{2} \mathrm{SO}_{4}\right)$, filtered, and concentrated to dryness. The crude extracts were analyzed by ESI-MS and TLC. The cell culture of S. lividans TK64/pHSA81 (control) [39] was used for negative control.

\section{Results}

\subsection{Construction and Metabolite Analysis of an srrO Mutant KA54}

The P450 monooxygenase gene $\mathrm{srrO}$ was inactivated to analyze its function in SRB biosynthesis. An apramycin resistance gene cassette was introduced into a 5 -terminal region of $\mathrm{srrO}$, and double crossover mutants were obtained through homologous recombination. Gene replacement was confirmed by Southern hybridization experiment. As shown in Figure S1, a 4.4-kb BspEI-XhoI fragment in parent 51252 was changed into two fragments with $2.3-\mathrm{kb}$ and $3.1-\mathrm{kb}$ in mutant KA54. A metabolite profile in strain KA54 was analyzed in comparison with that in parent 51252. KA54 produced lankacidin and lankamycin in a comparative level to 51252 (Figure 2A). There are two possibilities to explain that the disruption of the srrO showed no effect on the production of lankacidin and lankamycin; (1) SrrO is not involved in SRB biosynthesis, and (2) the signaling molecule(s) in the srrO mutant has(have) an ability to induce lankacidin and lankamycin production.

\subsection{Structural Elucidation of Signaling Molecules in KA54}

To investigate these possibilities mentioned above, we carried out the isolation of signaling molecules in KA54 (termed as KA54-SRBs). Cultivation of KA54 was stopped at a $36 \mathrm{~h}$ period to reduce the accumulation of lankacidin and lankamycin, which would disturb the SRB bioassay. A 30-L culture was extracted with ethyl acetate (EtOAc), and the resulting oil was purified by Sephadex LH20 with 
methanol. Each fraction was subjected to bioassay to check its antibiotic-inducing activity. The $s r r X$ mutant was used for an indicator strain that could restore lankacidin and lankamycin production in the presence of SRBs. The active fractions were combined and purified by series of silica gel chromatography with $\mathrm{CHCl}_{3}$-methanol $(50: 1, v / v)$ and toluene-EtOAc $(3: 1, v / v)$.

The active fractions were further analyzed by ESI-MS analysis. ESI-MS indicated the presence of two active components (KA54-SRB1 and KA54-SRB2) in the ratio 1:1 (Figure 2B). The molecular formulae for KA54-SRB1 and KA54-SRB2 were established to be $\mathrm{C}_{15} \mathrm{H}_{26} \mathrm{O}_{4}$ and $\mathrm{C}_{16} \mathrm{H}_{28} \mathrm{O}_{4}$, whose values were one oxygen smaller and two hydrogen larger when compared with those for SRB1 and SRB2, respectively. Due to the low amounts of active components KA54-SRB1 and KA54-SRB2, we further analyzed their structural assignments as a mixture (compound with $\mathrm{C}_{15} \mathrm{H}_{26} \mathrm{O}_{4}$ was termed as KA54-SRB1, while $\mathrm{C}_{16} \mathrm{H}_{28} \mathrm{O}_{4}$ as $\left.\mathrm{KA54}-\mathrm{SRB} 2\right)$.

The NMR spectra of KA54-SRBs were measured and compared with those of SRB1 and SRB2 in parent 51252. Based on ESI-MS analysis, one oxygen atom was replaced with two hydrogens in KA54-SRBs. In ${ }^{1} \mathrm{H}$ NMR of KA54-SRBs, three signals including a highly deshielded singlet methine proton at $\delta_{\mathrm{H}} 5.87$, a deshielded methine proton at $\delta_{\mathrm{H}} 4.48$, and a singlet methyl proton at $\delta_{\mathrm{H}} 2.07$ were conserved when compared with those in natural SRBs (Figure 2C), suggesting the presence of a 2-(1'-hydroxyl-alkyl)-3-methyl-4-hydroxybut-2-en-1,4-olide skeleton (red-color dashed blanket in Figure 2C). This butenolide skeleton was further confirmed by a 2D NMR technique including HMQC and HMBC spectra, which showed a good agreement with our previous report for natural SRBs [11]. Regarding the alkyl side chain branched at C-2, methylene proton signals at $\delta_{\mathrm{H}} 2.26$ (doublet), 2.18 (double-doublet), and 2.37-2.40 (multiplet) for C-5' and C-7' in natural SRBs could not be detected in KA54-SRBs (Figure 2C). Furthermore, carbonyl carbon at $\delta_{C} 213.1$ in natural SRBs could not be detected in HMBC and ${ }^{13} \mathrm{C}$ NMR spectra of KA54-SRBs, suggesting the replacement of C-6' ketone with methylene in KA54-SRBs. Thus, KA54-SRB1 and KA54-SRB2 were assigned to be 2-(1'-hydroxyl-8'-methylnonyl)-3-methyl-4-hydroxybut-2-en-1,4-olide (6'-deoxo-SRB1; compound 1) and 2-(1'-hydroxyl-8'-methyldecyl)-3-methyl-4-hydroxybut-2-en-1,4-olide (6'-deoxo-SRB2; compound 2) (Figure 1).

\subsection{Synthesis of $6^{\prime}$-deoxo-SRBS}

In order to confirm the proposed structures of $\mathbf{1}$ and $\mathbf{2}$, the $\left(1^{\prime} R\right)$-isomers (1a and $\left.\mathbf{2 a}\right)$ and the $\left(1^{\prime} S\right)$-isomers ( $\mathbf{1 b}$ and $\left.\mathbf{2} \mathbf{b}\right)$ were synthesized (Scheme 1$)$. The commercially available 1,6-hexanediol (3) was treated with 1 equivalent of $p$-toluenesulfonyl chloride to give monotosylate 4, which was then converted to tetrahydropyranyl ether 5 in 95\% yield. Cross coupling of compound 5 with Grignard reagent isobutylmagnesium bromide in the presence of $\mathrm{Li}_{2} \mathrm{CuCl}_{4}[40,41]$ generated a $\mathrm{C}_{10}$ unit, which was hydrolyzed with aqueous $\mathrm{HCl}$ to form isodecanol 7 in 91\% yield (2 steps). Alcohol 7 was subsequently oxidized with pyridinium chlorochromate (PCC) to give aldehyde 8 in $86 \%$ yield, which was then coupled with enantiomerically pure 3-methyl-4-(L-menthyloxy)but-2-en-1,4-olide (9) $[11,35,36]$ in the presence of lithium diisopropylamide (LDA) to give a diastereomeric mixture of 10a and $\mathbf{1 0 b}$ in the ratio $2: 1$ in $38 \%$ yield. They were separated by repeated runs of flash silica gel chromatography to obtain compounds $\mathbf{1 0 a}$ and $\mathbf{1 0 b}$ with over $95 \%$ diastereomeric excess based on the peak intensities of hemiacetal $\mathrm{H}-4$ proton signals $\left(\delta_{\mathrm{H}} 5.69\right.$ for 10a and $\delta_{\mathrm{H}} 5.71$ for $\left.10 \mathrm{~b}\right)$. In addition, the C-1' configuration was determined by the modified Mosher ester method [37]. These isomers 10a and 10b were separately treated with boron tribromide to form (1'R)-6'-deoxo-SRB1 (1a) and (1'S)-6'-deoxo-SRB1 (1b) in 79\% and 62\% yields, respectively.

$6^{\prime}$-deoxo-SRB2a (2a) and $6^{\prime}$-deoxo-SRB2b (2b) were synthesized in a similar manipulation for $\mathbf{1 a}$ and $\mathbf{1 b}$, except for a Grignard reagent, $(S)$-(2-methylbutyl)magnesium chloride, to form a $C_{11}$ unit 11 (Scheme 1B).

To confirm the C-1' stereochemistry in compounds $\mathbf{1}$ and $\mathbf{2}$, chiral HPLC analysis was performed (Figure 3). The retention times of $\mathbf{1}$ and $\mathbf{2}$ (21.1 and $53.3 \mathrm{~min}$, respectively) on a chiral HPLC column were identical to those of the synthetic $\left(1^{\prime} R\right)$-isomers $\mathbf{1 a}$ and $\mathbf{2 a}$, whereas the synthetic $\left(1^{\prime} S\right)$-isomers $\mathbf{1} \mathbf{b}$ 
and $\mathbf{2 b}$ eluted slightly earlier at 20.2 and $50.0 \mathrm{~min}$. Thus, the C-1' stereochemistry in 6'-deoxo-SRBs was the same as with SRBs.
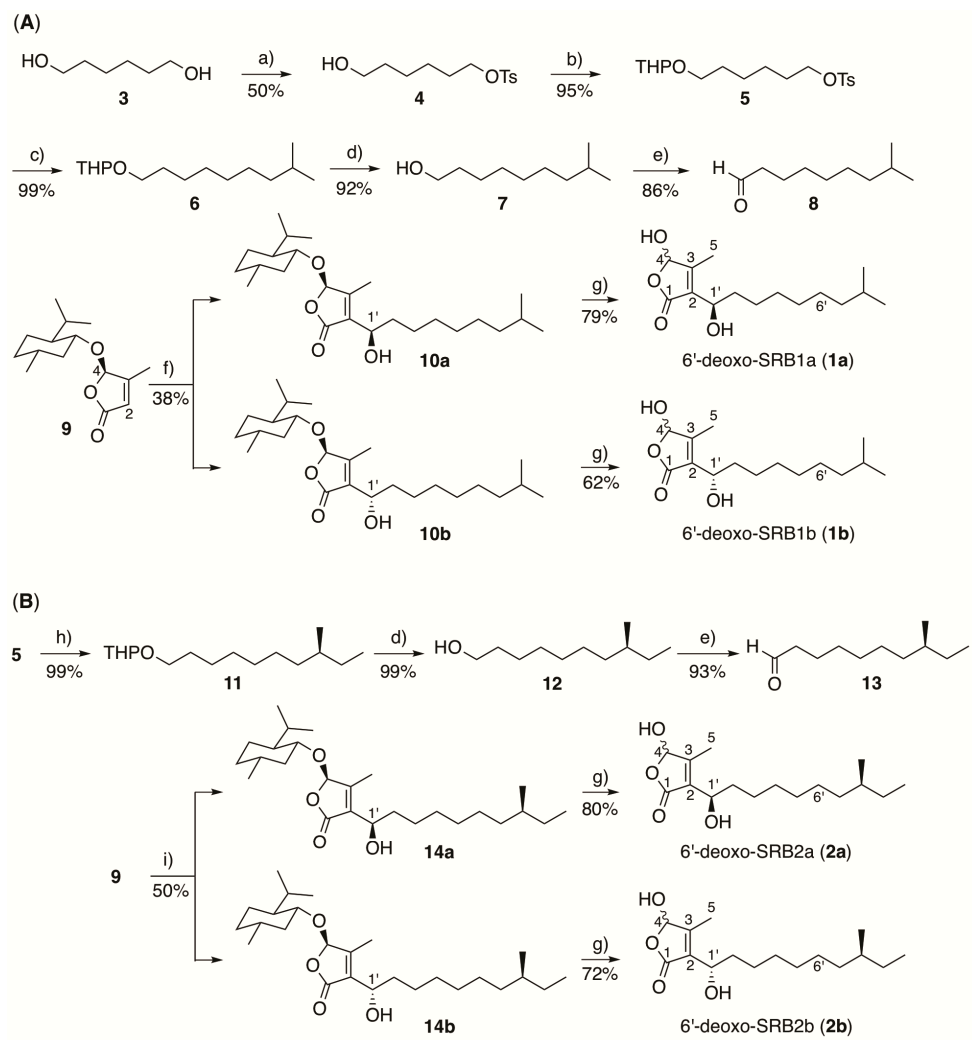

Scheme 1. Synthesis of SRB intermediates, (A) 6'-deoxo-SRB1 and (B) 6'-deoxo-SRB2. a) $p$-toluenesulfonyl chloride, $\mathrm{Et}_{3} \mathrm{~N}$, 4,4-dimethylaminopyridine, $\mathrm{CH}_{2} \mathrm{Cl}_{2} ;$ b) 3,4-dihydro-2H-pyran, p-toluenesulfonic acid, $\mathrm{CH}_{2} \mathrm{Cl}_{2} ; \mathrm{c}$ ) isobutylmagnesium bromide, $\mathrm{Li}_{2} \mathrm{CuCl}_{4}$, THF; d) $2 \mathrm{M} \mathrm{HCl}, \mathrm{THF}-\mathrm{MeOH}$ $(1: 1, v / v) ; \mathrm{e})$ pyridinium chlorochromate, $\left.\mathrm{CH}_{2} \mathrm{Cl}_{2} ; \mathrm{f}\right) \mathrm{LDA}$, THF-HMPA, and then 8; g) $\mathrm{BBr}_{3}, \mathrm{CH}_{2} \mathrm{Cl}_{2} ; \mathrm{h}$ ) (S)-(2-methylbutyl)magnesium chloride, $\mathrm{Li}_{2} \mathrm{CuCl}_{4}$, THF; i) LDA, THF-HMPA, and then 13.

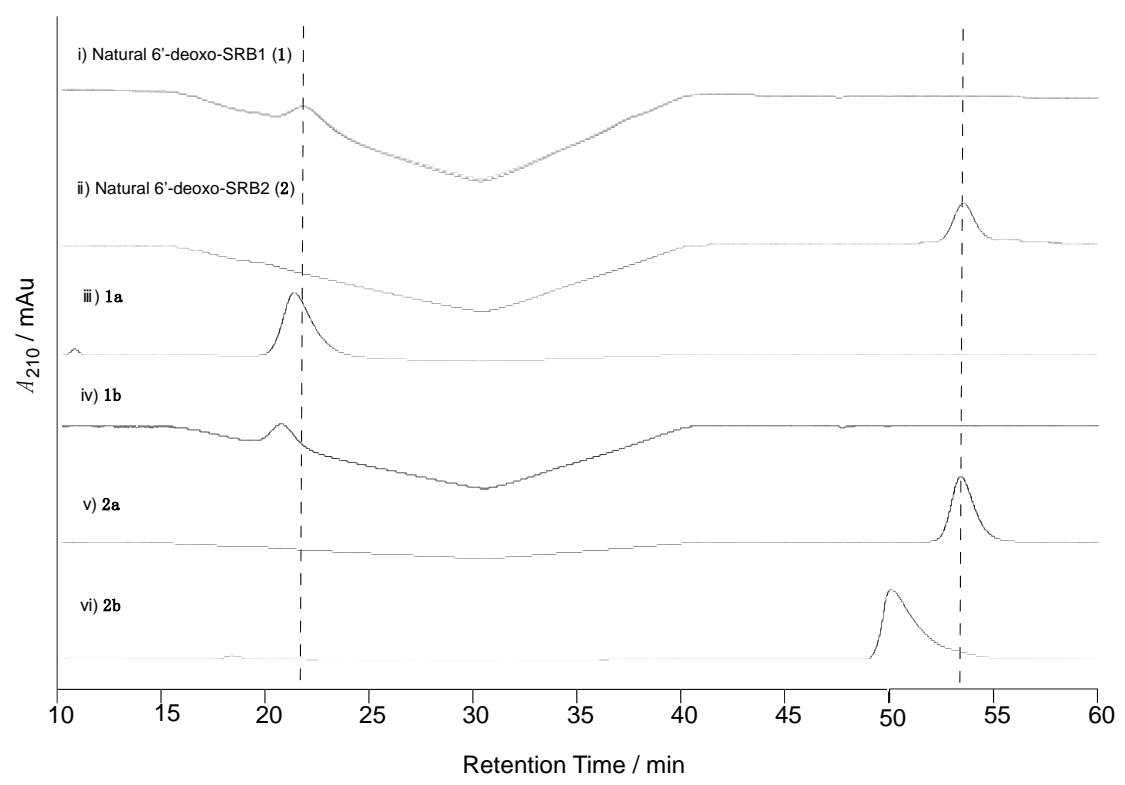

Figure 3. Determination of the C-1' configuration of natural $6^{\prime}$-deoxo-SRBs (1 and 2$)$ isolated from strain KA54. Chiral HPLC analysis of (i) natural 6'-deoxo-SRB1 (1), (ii) natural 6'-deoxo-SRB2 (2), (iii) synthetic 1a, (iv) $\mathbf{1 b}$, (v) 2a, and (vi) 2b. Elution profiles were monitored by UV absorbance at $210 \mathrm{~nm}$. 


\subsection{Ligand Affinity of $6^{\prime}$-deoxo-SRBs}

To compare an antibiotic-inducing activity of SRBs and 6'-deoxo-SRBs, a bioassay method using the srrX mutant was not suitable since $6^{\prime}$-deoxo-SRBs could be converted to SRBs in some extent by SrrO protein expressed in the srrX mutant. Therefore, the antibiotic-inducing activity of $6^{\prime}$-deoxo-SRBs was evaluated by minimum concentration to dissociate SrrA protein from a main target gene srrY, which encodes SARP and activates lankacidin and lankamycin production in S. rochei [22]. Gel shift assay was performed using a recombinant SrrA protein and a ${ }^{32} \mathrm{P}$-labeled DNA probe containing srrY-promoter (srrYp) region in the presence of either $6^{\prime}$-deoxo-SRB1 or SRB1 (Figure 4A). As shown in Figure 4B, minimum concentration of $6^{\prime}$-deoxo-SRB1 was 100-fold higher than that of SRB1. This finding indicated that C- $6^{\prime}$ keto group is important for improvement of antibiotic-inducing activity in S. rochei.

A

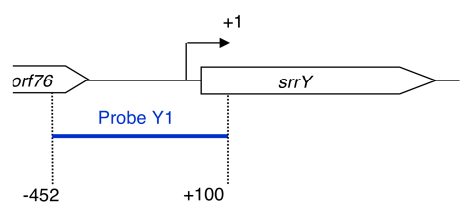

B (i)
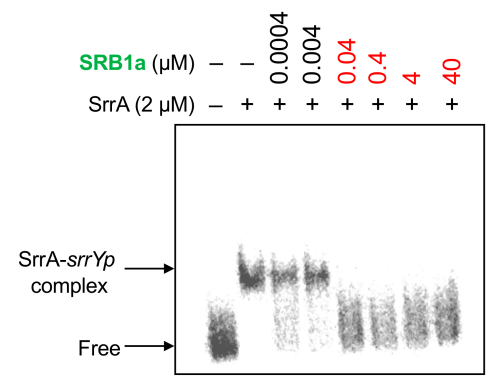

(ii)

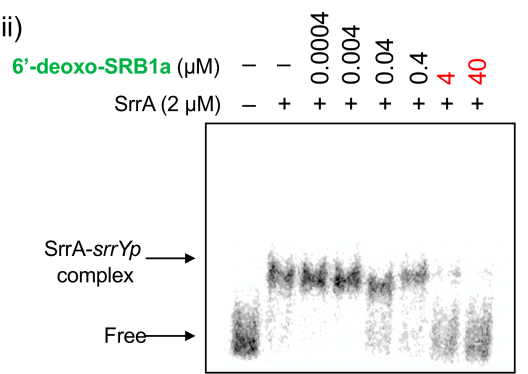

Figure 4. Gel shift assay of SrrA-binding in the upstream region of $\operatorname{srr} Y$. (A) Location of probe Y1 containing the $s r r Y$-promoter region $(s r r Y p)$. The description of probe preparation was described previously [22]. (B) Effect of the SRB1 (i) and 6'-deoxo-SRB1 (ii) on the binding of SrrA. Each reaction mixture contained $0.35 \mathrm{nM}$ probe $\mathrm{Y} 1$ and $2 \mu \mathrm{M}$ recombinant SrrA protein. To the reaction mixture, various concentrations of either synthetic SRB1 ((1'R)-isomer; Figure 1B) [11] or synthetic $6^{\prime}$-deoxo-SRB1 $\left(\left(1^{\prime} R\right)\right.$-isomer; Figure 1B) was added.

\subsection{Enzymatic Bioconversion of 6'-deoxo-SRBs by SrrO Protein}

To examine the enzymatic conversion of 6 '-deoxo-SRBs, we constructed SrrO expression system in Streptomyces. The $\mathrm{srrO}$ gene was cloned into pKAR3063H [23], a (His) ${ }_{6}$-tag derivative of streptomycete constitutive expression vector pHSA81 (Michihiko Kobayashi and Yoshiteru Hashimoto, personal communication), to afford pNTT01 (Figure S3A). This plasmid was transformed into the heterologous host, Streptomyces lividans TK64, to obtain the recombinant strain. Protein expression of (His) ${ }_{6}$-SrrO with $45-\mathrm{kDa}$ in size was confirmed by SDS-PAGE (Figure S3B). Most Streptomyces P450 enzymes associate with electron-recycling redox partners, ferredoxin/ferredoxin reductase, and they flexibly accept heterologous redox partners in other Streptomyces [42]. We therefore performed enzymatic bioconversion of $6^{\prime}$-deoxo-SRB1 (1) by the recombinant SrrO protein with the help of heterologous S. lividans redox partners. In an ESI-MS analysis (Figure 5A), two molecular ion peaks at $\mathrm{m} / \mathrm{z} 307$ 
and 309 were detected in the recombinant S. lividans TK64/pNTT01. In a TLC analysis (Figure 5B), compound $1(\mathrm{Rf}=0.8$ in hexane-EtOAc $=1: 2)$ was converted to two compounds in the recombinant S. lividans TK64/pNTT01 (lane 2); spot A showed a same Rf value with synthetic SRB1 (Rf = 0.5 in hexane-EtOAc $=1: 2$ ), while another spot B did lower $\mathrm{Rf}$ value $(\mathrm{Rf}=0.2$ in hexane-EtOAc $=1: 2)$. Time-dependent oxidation of compound 1 was observed in ESI-MS spectra (Figure 5C). Molecular ion peak at $293\left([\mathrm{M}+\mathrm{Na}]^{+}=\mathrm{C}_{15} \mathrm{H}_{26} \mathrm{O}_{4} \mathrm{Na}\right)$ for $\mathbf{1}$ diminished in a time-dependent manner (Panels i-vii), while that at $309\left([\mathrm{M}+\mathrm{Na}]^{+}=\mathrm{C}_{15} \mathrm{H}_{26} \mathrm{O}_{5} \mathrm{Na}\right)$ appeared as a major peak at the $1 \mathrm{~h}$ period (Panel ii). After $2 \mathrm{~h}$ periods, molecular ion peak at $307\left([\mathrm{M}+\mathrm{Na}]^{+}=\mathrm{C}_{15} \mathrm{H}_{24} \mathrm{O}_{5} \mathrm{Na}\right)$ corresponding to SRB1 became a major peak (Panel iii). Based on the C- $6^{\prime}$ oxidation degree in SRB biosynthesis, an intermediate with molecular ion peak at 309 was estimated to be $6^{\prime}$-deoxo-6'-hydroxy-SRB1 (Figure 5C), which possibly corresponded to spot B with lower Rf value on TLC (Figure 5B). Thus, SrrO converts 6'-deoxo-SRB1 into SRB1 via 6'-deoxo-6'-hydroxy-SRB1.

A

(i)

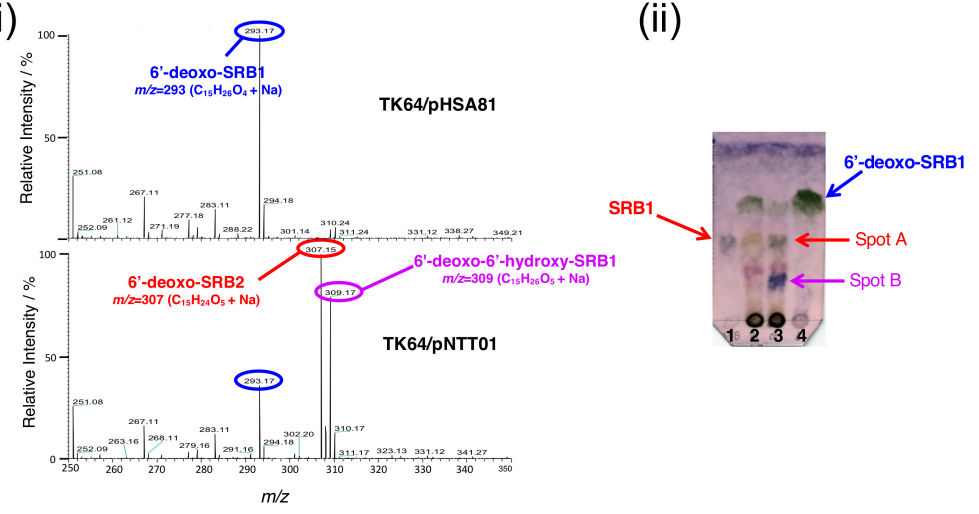

B

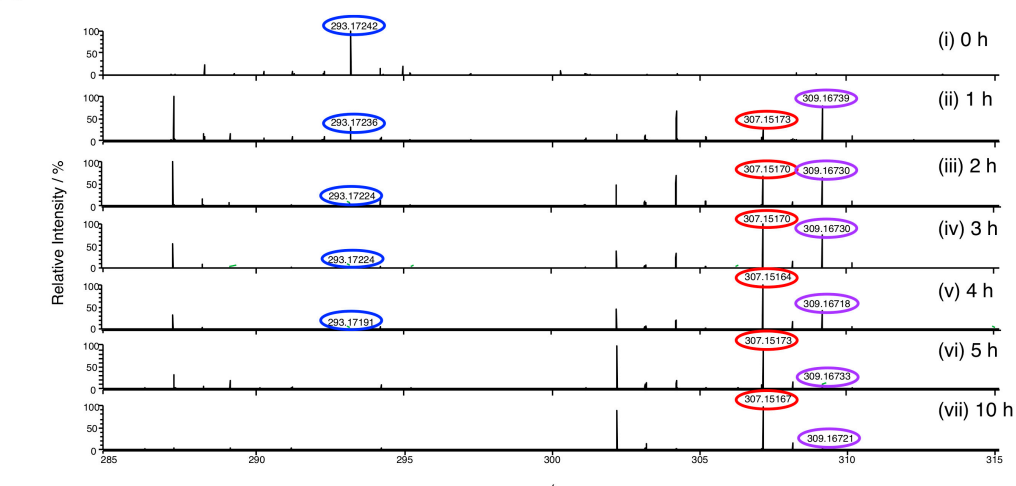

C

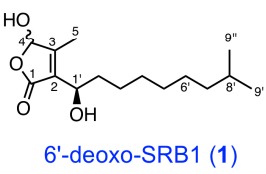

$m / z$

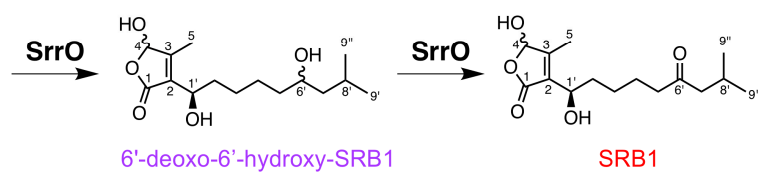

Figure 5. Enzymatic conversion of 6'-deoxo-SRB1 (1) by P450 monooxygenase SrrO in the Streptomyces lividans TK64/pNTT01 recombinant. (A) (i) ESI-MS spectra of reaction mixture in S. lividans TK64/pNTT01 (+SrrO) (upper panel) and S. lividans TK64/pHSA81 (control). (ii) TLC analysis of reaction mixture. Lane 1, the synthetic SRB1, lane 2, reaction mixture in S. lividans TK64/pHSA81; lane 3, reaction mixture in S. lividans TK64/pNTT01; lane 4, the synthetic $6^{\prime}$-deoxo-SRB1. TLC was developed with hexane-EtOAc $=1: 2(v / v)$, and baked after staining with anisaldehyde. (B) ESI-MS spectra of time-course enzymatic conversion of $6^{\prime}$-deoxo-SRB1. Samples were collected at $0,1,2,3,4,5$, and $10 \mathrm{~h}$ periods. (C) Scheme for two-stage oxidation of 6'-deoxo-SRB1 by SrrO. 
To judge the substrate preference on the $C-1^{\prime}$ configuration, enzymatic conversion was performed on a mixture of (1'R)-6'-deoxo-SRB1 (1a) and (1'S)-6'-deoxo-SRB1 (1b) (Figure 6). In chiral HPLC analysis, a slightly rapid decrease of peak intensity (around 1.3-fold) at $44.4 \mathrm{~min}$ for $\mathbf{1 b}$ was detected when compared with that at $45.1 \mathrm{~min}$ for 1a. This result indicated that SrrO slightly prefers unnatural $\left(1^{\prime} S\right)$-isomer $\mathbf{1 b}$ to a natural $\left(1^{\prime} R\right)$-isomer $\mathbf{1 a}$.

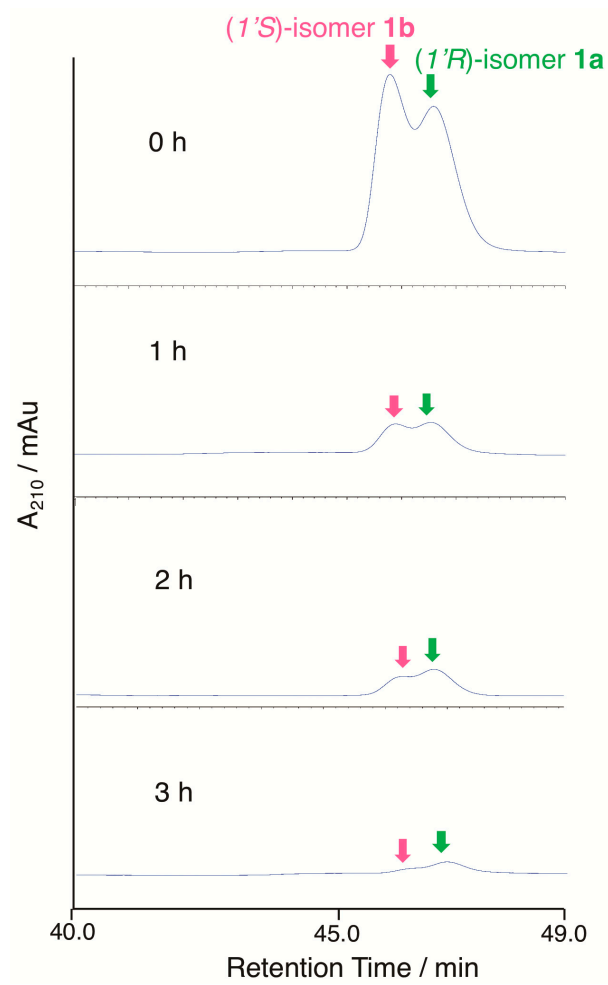

Figure 6. Substrate recognition of the C-1' stereochemistry by SrrO. Conversion efficiency of a mixture of $\left(1^{\prime} R\right)$-isomer $\mathbf{1 a}$ and $\left(1^{\prime} S\right)$-isomer $\mathbf{1} \mathbf{b}$ was analyzed by chiral HPLC. Chromatogram was monitored by UV absorbance at $210 \mathrm{~nm}$.

\section{Discussion}

The function of the P450 monooxygenase gene srrO in SRB biosynthesis was analyzed through gene disruption, gel-shift assay, and by in vivo enzymatic conversion. The srrO disruptant produced lankacidin and lankamycin in a comparative yield with the parent 51252, and accumulated novel signaling molecules, $6^{\prime}$-deoxo-SRB1 $(\mathbf{1}=\mathbf{1 a})$ and $6^{\prime}$-deoxo-SRB2 $(\mathbf{2}=\mathbf{2 a})$. Based on a ligand activity of signaling molecules for dissociation of their receptor SrrA, $6^{\prime}$-deoxo-SRB1 exhibits the 100-fold less binding activity compared with SRB1. Nevertheless, $6^{\prime}$-deoxo-SRBs could also bind to the specific SRB receptor SrrA to induce lankacidin and lankamycin production in S. rochei. At this stage, we have no answer why oxidation of C-6' methylene to ketone by SrrO took place at the final step in SRB biosynthesis. One of the plausible reasons is an increase of hydrophilic property in SRB to improve antibiotic-inducing activity. To our knowledge, this is a first report to obtain biosynthetic intermediates of signaling molecules in Streptomyces species.

SrrO protein catalyzes two-stage oxidation of $6^{\prime}$-deoxo-SRBs through $6^{\prime}$-deoxo- $6^{\prime}$-hydroxy-SRBs to form SRBs. The srrO recombinant converted $6^{\prime}$-deoxo-SRB1 1 to SRB1 in a time-dependent manner, whereas the control recombinant harboring empty vector pHSA81 was unable to oxidize 1 (Figure 5). In addition, biosynthetic intermediate $6^{\prime}$-deoxo-6' $6^{\prime}$-hydroxy-SRB1 could not be converted to SRB1 in the control recombinant S. lividans TK64/pHSA81 (data not shown). Hence, two-stage oxidation is enzymatically catalyzed by SrrO in SRB biosynthesis. SrrO could oxidize the C-6' methylene group on not only natural $\left(1^{\prime} R\right)$-isomers $\mathbf{1 a}$ but unnatural $\left(1^{\prime} S\right)$-isomers $\mathbf{1 b}$ (Figure 6$)$. This finding indicated that 
C-1' stereochemistry of SRBs is strictly controlled at an earlier biosynthetic step of SrrO, possibly by NAD-dependent dehydrogenase SrrG.

Several possible genes for SRB biosynthesis and antibiotic regulation were found around $\operatorname{srr} X$ (orf85) on pSLA2-L; the NAD-dependent dehydrogenase gene $\operatorname{srrG}$ (orf81), the phosphatase gene srrP (orf83), the P450 monooxygenase gene srrO (orf84), and the thioesterase gene srrH (orf86), together with repressor genes $\operatorname{sr} A$ (orf82) and $\operatorname{srB}$ (orf79). The possible biosynthetic pathway of SRBs were shown in Figure 7 based on the biosynthetic pathways for other signaling molecules, A-factor in S. griseus [8] and virginia butanolides in S. virginiae [43], and antifungal butenolide gladiofungin in Burkholderia gladioli HKI0739 [44]. Medium-chain $\left(\mathrm{C}_{12}\right.$ or $\left.\mathrm{C}_{13}\right) \beta$-keto acid was derived through fatty acid biosynthesis pathway; four units of malonyl CoA are condensed with either an isobutyryl CoA unit for SRB1 or a 2-methylbutyryl CoA unit for SRB2. These $\beta$-keto acids are condensed with a $\mathrm{C}_{3}$ unit (a hydrate form of glyceraldehyde 3-phosphate) by SrrX, then followed by spontaneous dephosphorylation, dihydroxylation, and intramolecular aldol condensation to generate the butenolide skeleton. The C-1' ketone moiety in butenolide intermediate will be reduced by dehydrogenase SrrG to synthesize 6'-deoxo-SRBs, which accept two-stage oxidation by SrrO to form SRBs.

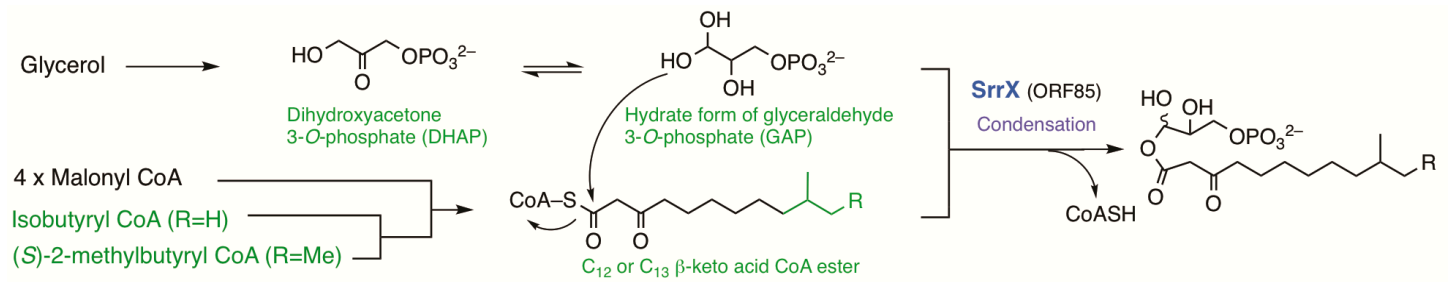

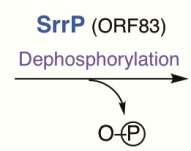
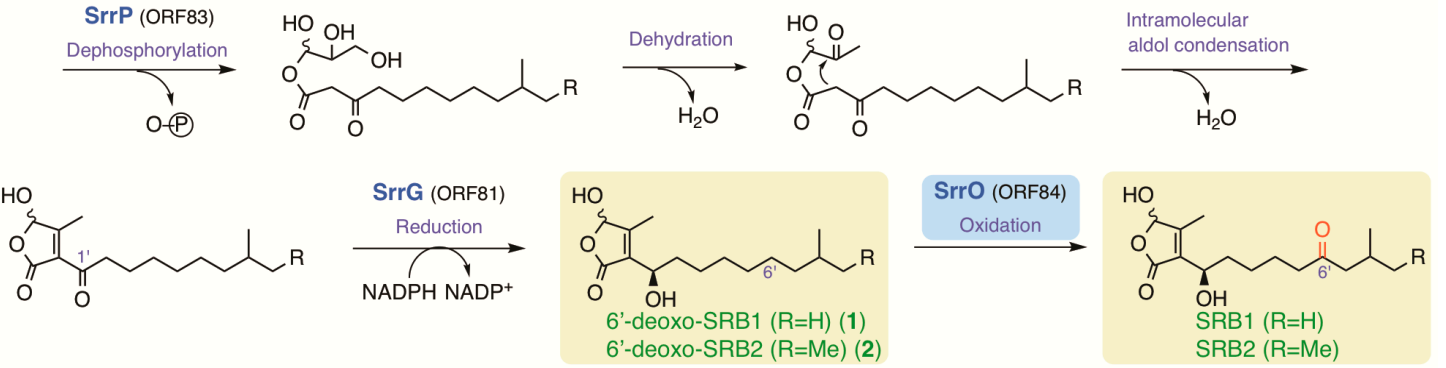

Figure 7. The possible biosynthetic pathway of SRBs in S. rochei. Me; methyl.

Streptomyces signaling molecules have a crucial role to induce secondary metabolite biosynthesis. Many Streptomyces strains have more than 30 secondary metabolites biosynthetic gene clusters in their genome; however, many of them (around 80-90\%) are silent in normal culture conditions. In S. rochei, 40 biosynthetic gene clusters ( 35 in the chromosome and 5 in pSLA2-L) are found; however, only six compounds are detected $[17,45]$. Although we do not yet know the reason why many of them are silent or poorly expressed, a lack of specific signaling molecules is one of a plausible possibility [46,47]. Streptomyces strains generally have multiple signaling-molecule receptor genes, suggesting that they may have a potential to recognize heterologous signaling molecules. Thus, signaling molecules may contribute as "genetic engineering-free" genome mining tools to act as communication signals between actinomycetes, between different bacteria, and/or between interkingdom.

\section{Conclusions}

We here investigated the role of a P450 monooxygenase gene srrO in SRB biosynthesis. Two signaling molecules in the srrO-disruptant KA54 were determined to be $6^{\prime}$-deoxo-SRB1 and 6 '-deoxo-SRB2 by ESI-MS, NMR, and HPLC analyses from $30 \mathrm{~L}$ culture extract. A ligand affinity of $6^{\prime}$-deoxo-SRB1 to SrrA was shown to be 100-fold less than that of SRB1. We further performed bioconversion of the synthetic $6^{\prime}$-deoxo-SRB1 in the S. lividans recombinant carrying SrrO-expression plasmid. Substrate $6^{\prime}$-deoxo-SRB1 was converted through $6^{\prime}$-deoxo- $6^{\prime}$-hydroxy-SRB1 to SRB1 in a 
time-dependent manner. Thus, these results clearly indicated that SrrO catalyzes the C- $6^{\prime}$ oxidation at a final step in SRB biosynthesis.

Supplementary Materials: The followings are available online at: http://www.mdpi.com/2218-273X/10/9/1237/s1. Table S1: Bacterial strains, plasmids, and oligonucleotides used in this study; Figure S1: Chemical structures of lankamycin, lankacidin C, lankacidinol A, and lankacidinol; Figure S2: Gene disruption of srrO (orf84); Figure S3: Overexpression of the SrrO protein; Figure S4: ${ }^{1} \mathrm{H}-\mathrm{NMR}$ of 6-hydroxyhexyl $p$-toluenesulfonate (4); Figure S5: ${ }^{13} \mathrm{C}$-NMR of 6-hydroxyhexyl $p$-toluenesulfonate (4); Figure S6: ${ }^{1} \mathrm{H}-\mathrm{NMR}$ of 6-((tetrahydro-2H-pyran-2-yl)oxy)hexyl p-toluenesulfonate (5); Figure S7: ${ }^{13} \mathrm{C}-\mathrm{NMR}$ of 6-((tetrahydro-2H-pyran-2-yl)oxy)hexyl p-toluenesulfonate (5); Figure S8: ${ }^{1} \mathrm{H}-\mathrm{NMR}$ of 2-((8-methylnonyl)oxy)tetrahydro-2H-pyran (6); Figure S9: ${ }^{13} \mathrm{C}-\mathrm{NMR}$ of 2-((8-methylnonyl)oxy)tetrahydro-2H-pyran (6); Figure S10: ${ }^{1} \mathrm{H}-\mathrm{NMR}$ of 8-menthylnonan-1-ol (7); Figure S11: ${ }^{13} \mathrm{C}-\mathrm{NMR}$ of 8-menthylnonan-1-ol (7); Figure S12: ${ }^{1} \mathrm{H}-\mathrm{NMR}$ of 8-menthylnonanal (8); Figure S13: ${ }^{13} \mathrm{C}-\mathrm{NMR}$ of 8-menthylnonanal (8); Figure S14: ${ }^{1} \mathrm{H}-\mathrm{NMR}$ of compound 10a; Figure S15: ${ }^{13} \mathrm{C}-\mathrm{NMR}$ of compound 10a; Figure S16: ${ }^{1} \mathrm{H}-\mathrm{NMR}$ of compound 10b; Figure S17: ${ }^{13} \mathrm{C}-\mathrm{NMR}$ of compound 10b; Figure S18: ${ }^{1} \mathrm{H}-\mathrm{NMR}$ of $6{ }^{\prime}$-deoxo-SRB1a (1a); Figure S19: ${ }^{13} \mathrm{C}-\mathrm{NMR}$ of 6 -deoxo-SRB1a (1a); Figure S20: ${ }^{1} \mathrm{H}-\mathrm{NMR}$ of $6{ }^{\prime}$-deoxo-SRB1b (1b); Figure S21: ${ }^{13} \mathrm{C}-\mathrm{NMR}$ of 6'-deoxo-SRB1b (1b); Figure S22: ${ }^{1} \mathrm{H}-\mathrm{NMR}$ of 2-((S)-8-methyldecyl)oxy)tetrahydro-2H-pyran (11); Figure S23: ${ }^{13} \mathrm{C}-\mathrm{NMR}$ of 2-(((S)-8-methyldecyl)oxy)tetrahydro-2H-pyran (11); Figure S24: ${ }^{1} \mathrm{H}-\mathrm{NMR}$ of $(S)-8$-methyldecan-1-ol (12); Figure S25: ${ }^{13} \mathrm{C}-\mathrm{NMR}$ of (S)-8-methyldecan-1-ol (12); Figure S26: ${ }^{1} \mathrm{H}-\mathrm{NMR}$ of (S)-8-methyldecanal (13); Figure S27: ${ }^{13} \mathrm{C}-\mathrm{NMR}$ of $(S)-8-$ methyldecanal (13); Figure S28: ${ }^{1} \mathrm{H}-\mathrm{NMR}$ of compound 14a; Figure S29: ${ }^{13} \mathrm{C}-\mathrm{NMR}$ of compound 14a; Figure S30: ${ }^{1} \mathrm{H}-\mathrm{NMR}$ of compound 14b; Figure S31: ${ }^{13} \mathrm{C}-\mathrm{NMR}$ of compound 14b; Figure S32: ${ }^{1} \mathrm{H}-\mathrm{NMR}$ of $6^{\prime}$-deoxo-SRB2a (2a); Figure S33: ${ }^{13} \mathrm{C}-\mathrm{NMR}$ of $6^{\prime}$-deoxo-SRB2a (2a); Figure S34: ${ }^{1} \mathrm{H}-\mathrm{NMR}$ of $6^{\prime}$-deoxo-SRB2b (2b); Figure S35: ${ }^{13} \mathrm{C}-\mathrm{NMR}$ of $66^{\prime}$-deoxo-SRB2b (2b).

Author Contributions: A.T., N.H., N.T., and K.A. designed the experiments, A.T., N.H., N.T., and K.A. performed the experiments, A.T., N.H., N.T., and K.A. analyzed the data, and A.T. and K.A. wrote the manuscript with input from all of the authors. All authors have read and agreed to the published version of the manuscript.

Funding: This work was supported by Grants-in-Aid for Scientific Research on Innovative Areas (23108515, 25108718, 17H05446, and 19H04659 to K.A.) from Ministry of Education, Culture, Sports, Science, and Technology of Japan (MEXT), Grants-in-Aid for Scientific Research (B) (16H04917 to K.A.) and for Challenging Exploratory Research (16K14915 to K.A.) from the Japan Society for the Promotion of Science (JSPS). This work was partly supported by a JSPS A3 Foresight Program (to A.T. and K.A.).

Acknowledgments: We are grateful to Tomoko Amimoto (Natural Science Center for Basic Research and Development (N-BARD), Hiroshima University) for measurement of the high-resolution mass spectra, and Kuninobu Inada (N-BARD, Hiroshima University) for his help in radioisotope experiments. We thank Michihiko Kobayashi and Yoshiteru Hashimoto (Tsukuba University) for providing constitutive expression vector pHSA81.

Conflicts of Interest: The authors declare no conflict of interest.

\section{References}

1. Bibb, M.J. Regulation of secondary metabolism in streptomycetes. Curr. Opin. Microbiol. 2005, 8, $208-215$. [CrossRef] [PubMed]

2. Horinouchi, S.; Beppu, T. Hormonal control by A-factor of morphological development and secondary metabolism in Streptomyces. Proc. Jpn. Acad. Ser. B 2007, 83, 277-295. [CrossRef]

3. Takano, E. Gamma-butyrolactones: Streptomyces signalling molecules regulating antibiotic production and differentiation. Curr. Opin. Microbiol. 2006, 9, 287-294. [CrossRef] [PubMed]

4. Niu, G.; Chater, K.F.; Tian, Y.; Zhang, J.; Tan, H. Specialized metabolites regulating antibiotic biosynthesis in Streptomyces spp. FEMS Microbiol. Rev. 2016, 40, 554-573. [CrossRef] [PubMed]

5. Arakawa, K. Manipulation of metabolic pathway controlled by signaling molecules, inducers of antibiotic production, for genome mining in Streptomyces spp. Antonie Van Leeuwen. 2018, 111, 743-751. [CrossRef] [PubMed]

6. Kong, D.; Wang, X.; Nie, J.; Niu, G. Regulation of antibiotic production by signaling molecules in Streptomyces. Front. Microbiol. 2019, 10, 2927. [CrossRef] [PubMed]

7. Hara, O.; Beppu, T. Mutants blocked in streptomycin production in Streptomyces griseus-the role of A-factor. J. Antibiot. 1982, 35, 349-358. [CrossRef] [PubMed] 
8. Kato, J.; Funa, N.; Watanabe, H.; Ohnishi, Y.; Horinouchi, S. Biosynthesis of gamma-butyrolactone autoregulators that switch on secondary metabolism and morphological development in Streptomyces. Proc. Natl. Acad. Sci. USA 2007, 104, 2378-2383. [CrossRef]

9. Corre, C.; Song, L.; O'Rourke, S.; Chater, K.F.; Challis, G.L. 2-Alkyl-4-hydroxymethylfuran-3-carboxylic acids, antibioticproduction inducers discovered by Streptomyces coelicolor genome mining. Proc. Natl. Acad. Sci. USA 2008, 105, 17510-17515. [CrossRef] [PubMed]

10. Kitani, S.; Miyamoto, K.T.; Takamatsu, S.; Herawati, E.; Iguchi, H.; Nishitomi, K.; Uchida, M.; Nagamitsu, T.; Omura, S.; Ikeda, H.; et al. Avenolide, a Streptomyces hormone controlling antibiotic production in Streptomyces avermitilis. Proc. Natl. Acad. Sci. USA 2011, 108, 16410-16415. [CrossRef]

11. Arakawa, K.; Tsuda, N.; Taniguchi, A.; Kinashi, H. The butenolide signaling molecules SRB1 and SRB2 induce lankacidin and lankamycin production in Streptomyces rochei. ChemBioChem 2012, 13, 1447-1457. [CrossRef] [PubMed]

12. Kinashi, H.; Mori, E.; Hatani, A.; Nimi, O. Isolation and characterization of large linear plasmids from lankacidin-producing Streptomyces species. J. Antibiot. 1994, 47, 1447-1455. [CrossRef] [PubMed]

13. Auerbach, T.; Mermershtain, I.; Davidovich, C.; Bashan, A.; Belousoff, M.; Wekselman, I.; Zimmerman, E.; Xiong, L.; Klepacki, D.; Arakawa, K.; et al. The structure of ribosome-lankacidin complex reveals ribosomal sites for synergistic antibiotics. Proc. Natl. Acad. Sci. USA 2010, 107, 1983-1988. [CrossRef] [PubMed]

14. Belousoff, M.J.; Shapira, T.; Bashan, A.; Zimmerman, E.; Rozenberg, H.; Arakawa, K.; Kinashi, H.; Yonath, A. Crystal structure of the synergistic antibiotic pair, lankamycin and lankacidin, in complex with the large ribosomal subunit. Proc. Natl. Acad. Sci. USA 2011, 108, 2717-2722. [CrossRef] [PubMed]

15. Ayoub, A.T.; El-Magd, R.M.A.; Xiao, J.; Lewis, C.W.; Tilli, T.M.; Arakawa, K.; Nindita, Y.; Chan, G.; Sun, L.; Glover, M.; et al. Antitumor activity of lankacidin antibiotics is due to microtubule stabilization via a paclitaxel-like mechanism. J. Med. Chem. 2016, 59, 9532-9540. [CrossRef] [PubMed]

16. Ayoub, A.T.; Elrefaiy, M.A.; Arakawa, K. Computational prediction of the mode of binding of antitumor lankacidin C to tubulin. ACS Omega. 2019, 4, 4461-4471. [CrossRef] [PubMed]

17. Mochizuki, S.; Hiratsu, K.; Suwa, M.; Ishii, T.; Sugino, F.; Yamada, K.; Kinashi, H. The large linear plasmid pSLA2-L of Streptomyces rochei has an unusually condensed gene organization for secondary metabolism. Mol. Microbiol. 2003, 48, 1501-1510. [CrossRef]

18. Arakawa, K.; Sugino, F.; Kodama, K.; Ishii, T.; Kinashi, H. Cyclization Mechanism for the Synthesis of Macrocyclic Antibiotic Lankacidin in Streptomyces rochei. Chem. Biol. 2005, 12, 249-256. [CrossRef]

19. Arakawa, K.; Kodama, K.; Tatsuno, S.; Ide, S.; Kinashi, H. Analysis of the loading and hydroxylation steps in lankamycin biosynthesis in Streptomyces rochei. Antimicrob. Agents Chemother. 2006, 50, 1946-1952. [CrossRef]

20. Arakawa, K. Genetic and biochemical analysis of the antibiotic biosynthetic gene clusters on the Streptomyces linear plasmid. Biosci. Biotechnol. Biochem. 2014, 78, 183-189. [CrossRef] [PubMed]

21. Arakawa, K.; Mochizuki, S.; Yamada, K.; Noma, T.; Kinashi, H. Gamma-Butyrolactone autoregulator-receptor system involved in lankacidin and lankamycin production and morphological differentiation in Streptomyces rochei. Microbiology 2007, 153, 1817-1827. [CrossRef] [PubMed]

22. Yamamoto, S.; He, Y.; Arakawa, K.; Kinashi, H. Gamma-Butyrolactone-dependent expression of the SARP gene srrY plays a central role in the regulatory cascade leading to lankacidin and lankamycin production in Streptomyces rochei. J. Bacteriol. 2008, 190, 1308-1316. [CrossRef] [PubMed]

23. Suzuki, T.; Mochizuki, S.; Yamamoto, S.; Arakawa, K.; Kinashi, H. Regulation of lankamycin biosynthesis in Streptomyces rochei by two SARP genes, srrY and srrZ. Biosci. Biotechnol. Biochem. 2010, 74, 819-827. [CrossRef] [PubMed]

24. Lamb, D.C.; Waterman, M.R.; Kelly, S.L.; Guengerich, F.P. Cytochromes P450 and drug discovery. Curr. Opin. Biotechnol. 2007, 18, 504-512. [CrossRef] [PubMed]

25. Pylypenko, O.; Schlichting, I. Structural aspects of ligand binding to and electron transfer in bacterial and fungal P450s. Annu. Rev. Biochem. 2004, 73, 991-1018. [CrossRef]

26. Rudolf, J.D.; Chang, C.Y.; Ma, M.; Shen, B. Cytochromes P450 for natural product biosynthesis in Streptomyces: Sequence, structure, and function. Nat. Prod. Rep. 2017, 34, 1141-1172. [CrossRef]

27. Rudi, F. Tuning P450 Enzymes as Oxidation Catalysts. ACS Catal. 2012, 2, 647-666. 
28. Haydock, S.F.; Dowson, J.A.; Dhillon, N.; Roberts, G.A.; Cortes, J.; Leadlay, P.F. Cloning and sequence analysis of genes involved in erythromycin biosynthesis in Saccharopolyspora erythraea: Sequence similarities between EryG and a family of S-adenosylmethionine-dependent methyltransferases. Mol. Gen. Genet. 1991, 230, 120-128. [CrossRef] [PubMed]

29. Stassi, D.; Donadio, S.; Staver, M.J.; Katz, L. Identification of a Saccharopolyspora erythraea gene required for the final hydroxylation step in erythromycin biosynthesis. J. Bacteriol. 1993, 175, 182-189. [CrossRef] [PubMed]

30. Rodriguez, A.M.; Olano, C.; Méndez, C.; Hutchinson, C.R.; Salas, J.A. A cytochrome P450-like gene possibly involved in oleandomycin biosynthesis by Streptomyces antibioticus. FEMS Microbiol. Lett. 1995, 127, 117-120. [CrossRef]

31. Kieser, T.; Bibb, M.J.; Buttner, M.J.; Chater, K.F.; Hopwood, D.A. Practical Streptomyces Genetics; The John Innes Foundation: Norwich, UK, 2000.

32. Zhang, H.; Shinkawa, H.; Ishikawa, J.; Kinashi, H.; Nimi, O. Improvement of transformation system in Streptomyces using a modified regeneration medium. J. Ferment. Bioeng. 1997, 83, 217-221. [CrossRef]

33. Sambrook, J.; Fritsch, E.F.; Maniatis, T. Molecular Cloning: A Laboratory Manual; Cold Spring Harbor Laboratory Press: Cold Spring Harbor, NY, USA, 1989.

34. Ishikawa, J.; Niino, Y.; Hotta, K. Construction of pRES18 and pRES19, Streptomyces-Escherichia coli shuttle vectors carrying multiple cloning sites. FEMS Microbiol. Lett. 1996, 145, 113-116. [CrossRef]

35. Bourguignon, J.J.; Wermuth, C.G. Lactone chemistry. Synthesis of $\beta$-substituted, $\gamma$-functionalized Butanolides and Butenolides and Succinaldehydic Acids from Glyoxylic Acid. J. Org. Chem. 1981, 46, 4889-4894. [CrossRef]

36. Feringa, B.L.; de Lange, B.; de Jong, J.C. Synthesis of enantiomerically pure $\gamma$-(menthyloxy) butenolides and (R)- and (S)-2-methyl-1,4-butanediol. J. Org. Chem. 1989, 54, 2471-2475. [CrossRef]

37. Ohtani, I.; Kusumi, T.; Kashman, Y.; Kakisawa, H. High-field FT NMR application of Mosher's method. The absolute configurations of marine terpenoids. J. Am. Chem. Soc. 1991, 113, 4092-4096. [CrossRef]

38. Misaki, Y.; Yamamoto, S.; Suzuki, T.; Iwakuni, M.; Sasaki, H.; Takahashi, Y.; Inada, K.; Kinashi, H.; Arakawa, K. $\mathrm{SrrB}$, a pseudo-receptor protein, acts as a negative regulator for lankacidin and lankamycin production in Streptomyces rochei. Front. Microbiol. 2020, 11, 1089. [CrossRef] [PubMed]

39. Yamauchi, Y.; Nindita, Y.; Hara, K.; Umeshiro, A.; Yabuuchi, Y.; Suzuki, T.; Kinashi, H.; Arakawa, K. Quinoprotein dehydrogenase functions at the final oxidation step of lankacidin biosynthesis in Streptomyces rochei 7434AN4. J. Biosci. Bioeng. 2018, 126, 145-152. [CrossRef]

40. Tamura, M.; Kochi, J. Coupling of Grignard reagents with organic halides. Synthesis 1971, 3, 303-305. [CrossRef]

41. Arakawa, K.; Eguchi, T.; Kakinuma, K. Specific deuterium labeling of archaeal 36-membered macrocyclic diether lipid. Bull. Chem. Soc. Jpn. 1998, 71, 2419-2426. [CrossRef]

42. Hussain, H.A.; Ward, J.M. Enhanced heterologous expression of two Streptomyces griseolus cytochrome P450s and Streptomyces coelicolor ferredoxin reductase as potentially efficient hydroxylation catalysts. Appl. Environ. Microbiol. 2003, 69, 373-382. [CrossRef] [PubMed]

43. Sakuda, S.; Higashi, A.; Tanaka, S.; Nihira, T.; Yamada, Y. Biosynthesis of virginiae butanolide, a butyrolactone autoregulator from Streptomyces. J. Am. Chem. Soc. 1992, 114, 663-668. [CrossRef]

44. Niehs, S.P.; Kumpfmuüller, J.; Dose, B.; Little, R.F.; Ishida, K.; Florez, L.V.; Kaltenpoth, M.; Hertweck, C. Insect-associated bacteria assemble the antifungal butenolide gladiofungin by non-canonical polyketide chain termination. Angew. Chem. Int. Ed. Engl. 2020; in press. [CrossRef]

45. Nindita, Y.; Cao, Z.; Fauzi, A.A.; Teshima, A.; Misaki, Y.; Muslimin, R.; Yang, Y.; Shiwa, Y.; Yoshikawa, H.; Tagami, M.; et al. The genome sequence of Streptomyces rochei 7434AN4, which carries a linear chromosome and three characteristic linear plasmids. Sci. Rep. 2019, 9, 10973. [CrossRef] [PubMed]

46. Liu, G.; Chater, K.F.; Chandra, G.; Niu, G.; Tan, H. Molecular regulation of antibiotic biosynthesis in Streptomyces. Microbiol. Mol. Biol. Rev. 2013, 77, 112-143. [CrossRef] [PubMed]

47. Martín, J.F.; Liras, P. Harnessing microbiota interactions to produce bioactive metabolites: Communication signals and receptor proteins. Curr. Opin. Pharmacol. 2019, 48, 8-16. [CrossRef] [PubMed]

(C) 2020 by the authors. Licensee MDPI, Basel, Switzerland. This article is an open access article distributed under the terms and conditions of the Creative Commons Attribution (CC BY) license (http://creativecommons.org/licenses/by/4.0/). 